\title{
Rotation and Magnetic Field Effect on Surface Waves Propagation in an Elastic Layer Lying over a Generalized Thermoelastic Diffusive Half-Space with Imperfect Boundary
}

\author{
S. M. Abo-Dahab, ${ }^{1,2}$ Kh. Lotfy, ${ }^{3}$ and A. Gohaly ${ }^{1}$ \\ ${ }^{1}$ Mathematics Department, Faculty of Science, Taif University, Taif 888, Saudi Arabia \\ ${ }^{2}$ Mathematics Department, Faculty of Science, SVU, Qena 83523, Egypt \\ ${ }^{3}$ Mathematics Department, Faculty of Science, Zagazig University, Zagazig 44519, Egypt \\ Correspondence should be addressed to Kh. Lotfy; khlotfy_1@yahoo.com
}

Received 7 April 2014; Accepted 14 October 2014

Academic Editor: Gongnan Xie

Copyright ( $) 2015$ S. M. Abo-Dahab et al. This is an open access article distributed under the Creative Commons Attribution License, which permits unrestricted use, distribution, and reproduction in any medium, provided the original work is properly cited.

\begin{abstract}
The aim of the present investigation is to study the effects of magnetic field, relaxation times, and rotation on the propagation of surface waves with imperfect boundary. The propagation between an isotropic elastic layer of finite thickness and a homogenous isotropic thermodiffusive elastic half-space with rotation in the context of Green-Lindsay (GL) model is studied. The secular equation for surface waves in compact form is derived after developing the mathematical model. The phase velocity and attenuation coefficient are obtained for stiffness, and then deduced for normal stiffness, tangential stiffness and welded contact. The amplitudes of displacements, temperature, and concentration are computed analytically at the free plane boundary. Some special cases are illustrated and compared with previous results obtained by other authors. The effects of rotation, magnetic field, and relaxation times on the speed, attenuation coefficient, and the amplitudes of displacements, temperature, and concentration are displayed graphically.
\end{abstract}

\section{Introduction}

The foundations of magnetoelasticity were presented by Knopoff [1] and Chadwick [2] and developed by Kaliski and Petykiewicz [3]. An increasing attention is devoted to the interaction between magnetic field and strain field in a thermoelastic solid due to its many applications in the fields of geophysics, plasma physics, and related topics. All papers quoted above assumed that the interactions between the two fields take place by means of the Lorentz forces appearing in the equations of motion and by means of a term entering Ohm's law and describing the electric field produced by the velocity of a material particle, moving in a magnetic field. The most ideal interface model, as it is known, is called perfect bond interface where the displacement and traction are continuous across the interface. However, interfaces are seldom perfect. Therefore, various imperfect models such as three-phase and linear models like spring models have been introduced by Yu et al. [4], Yu [5], and Benveniste [6]. Perhaps the most frequently studied imperfect interface model is the smooth bond interface, where the normal components of the displacements and traction are continuous across the interface, while the shear traction components are zero on the interface. Lord and Shulman [7] formulated a generalized theory of thermoelasticity with one thermal relaxation time, who obtained a wave equation by postulating a new law of heat conduction instead of classical Fourier's law. Green and Lindsay [8] developed a temperature rate-dependent thermoelasticity that includes two thermal relaxation times and does not violate the classical Fourier's law of heat conduction, when the body under consideration has a center of symmetry. Hetnarski and Ignaczak [9] introduced a review and presentation of generalized theories of thermoelasticity. Diffusion can be defined as the random walk of an assemble of particles from regions of high concentration to that of low concentration. Nowadays, there is a great deal of interest in 
the study of phenomena due to its applications in geophysics and electronic industry. In integrated circuit fabrication, diffusion is used to introduce "depants" in controlled amounts into semiconductor substance. In particular, diffusion is used to form the base and emitter in bipolar transistors, integrated resistors, and the source/drain in metal oxide semiconductor (MOS) transistors and polysilicon gates in MOS transistors. In most of the applications, the concentration is calculated using Fick's law. This is simple law which does not take into consideration the mutual interaction between the introduced substance and the medium into which introduced. Study of the diffusion phenomenon is used to improve the conditions of oil extractions. These days' oil companies are interested in the process of thermoelastic diffusion for more efficient extraction of oil from oil deposits. Until recently, thermodiffusion in solids, especially in metals, was considered as a quantity that is independent of body deformation. Practice, however, indicates that the process of thermodiffusion could have a very considerable influence on the deformation of the body. Thermodiffusion in elastic solid is due to the coupling of temperature, mass diffusion, and strain in addition to the exchange of heat and mass with the environment. Nowacki [10-13] developed the theory of thermoelastic diffusion by using coupled thermoelastic model. This implies infinite speed of propagation of thermoelastic waves. Olesiak and Pyryev [14] investigated the theory of thermoelastic diffusion and coupled quasistationary problems of thermal diffusion for an elastic layer. They studied the influence of cross effects arising from the coupling of the fields of temperature, mass diffusion, and strain due to which the thermal excitation results in additional mass concentration and generates additional fields of temperature. Sherief et al. [15] developed the generalized theory of thermoelastic diffusion with one relaxation time which allows finite speeds of propagation of waves.

Recently, Sherief and Saleh [16] investigated the problem of a thermoelastic half-space in the context of the theory of generalized thermoelastic diffusion with one relaxation time. Singh [17] discussed the reflection phenomena of waves from free surface of a thermoelastic diffusion with one relaxation time and with two relaxation times in [18]. Aouadi [19-21] investigated different problems in thermoelastic diffusion. Sharma and Walia $[22,23]$ discussed the effect of rotation on Rayleigh waves in the piezothermoelastic half-space. Kumar and Kansal [24] discussed the propagation of Rayleigh waves on free surface in transversely isotropic thermoelastic diffusion. Kumar and Kansal [25] derived the basic equations for generalized thermoelastic diffusion and discussed the Lamb waves. Dawn and Chakraborty [26] studied Rayleigh waves in Green-Lindsay's model of generalized thermoelastic media. Kumar and Chawla [27] investigated the effect of rotation and stiffness on surface waves propagation in an elastic layer lying over a generalized thermodiffusive elastic halfspace with imperfect boundary. New contributions on waves propagation in thermoelastic media have been discussed [2832].

In this paper, linear model is adopted to represent the imperfectly bonded interface conditions. The linear model is simplified and idealized situation of imperfectly bonded interface, where the discontinuities in displacements at interfaces have a linear relationship with the interface stresses. Taking these applications into account, the surface waves propagation at imperfect boundary between an isotropic elastic layer and isotropic thermodiffusive elastic half-space with rotation in the context of Green-Lindsay theory is investigated. The phase velocity and attenuation coefficients of wave propagation have been computed from the secular equations. The amplitudes of displacements, temperature, concentration, and specific loss are computed and depicted graphically to make clear the influence of magnetic field, rotation, stiffness, relaxation times, and diffusion on the phenomena and compare with the practical results.

\section{Basic Equations}

The basic governing equations for homogenous generalized thermodiffusive solid in the absence of heat and mass diffusion sources are as follows (Singh [17]).

(i) Constitutive relations are

$$
\sigma_{j i}=2 \mu \varepsilon_{j i}+\left[\lambda \varepsilon_{k k}-\beta_{1}\left(T+\tau_{1} \dot{T}\right)-\beta_{2}\left(C+\tau^{\prime} \dot{C}\right)\right] \delta_{j i} .
$$

(ii) Equation of motion in the rotating frame of reference is

$$
\begin{aligned}
\mu u_{i, j j} & +(\lambda+\mu) u_{j, i j}-\beta_{1}\left(T+\tau_{1} \dot{T}\right)_{, i} \\
- & \beta_{2}\left(C+\tau^{\prime} \dot{C}\right)_{, i}+F_{i} \\
& =\rho[\ddot{\vec{u}}+\vec{\Omega} \times \vec{\Omega} \times \vec{u}+2 \vec{\Omega} \times \dot{\vec{u}}]_{i},
\end{aligned}
$$

where $\vec{\Omega} \times \vec{\Omega} \times \vec{u}$ is the centripetal acceleration due to the time varying motion only and $2 \vec{\Omega} \times \overrightarrow{\vec{\Omega}}$ is the Coriolis acceleration:

$$
\begin{gathered}
\vec{\Omega} \times \vec{\Omega} \times \vec{u}=\left(-\Omega^{2} u, 0,-\Omega^{2} w\right)=-\Omega^{2}(u, 0, w) \\
2 \vec{\Omega} \times \dot{\vec{u}}=2(\Omega \dot{w}, 0,-\Omega \dot{u})=2 \Omega(\dot{w}, 0,-\dot{u}),
\end{gathered}
$$

where

$$
\vec{F}=\vec{J} \times \vec{B}
$$

Consider that the medium is a perfect electric conductor; we take the linearized Maxwell equations governing the electromagnetic field, taking into account absence of the displacement current (SI):

$$
\begin{gathered}
\operatorname{curl} \vec{h}=\vec{J}, \quad \operatorname{curl} \vec{E}=-\mu_{e} \frac{\partial \vec{h}}{\partial t}, \\
\operatorname{div} \vec{h}=0, \quad \operatorname{div} \vec{E}=0,
\end{gathered}
$$

where

$$
\vec{h}=\operatorname{curl}\left(\vec{u} \times \overrightarrow{H_{0}}\right)
$$



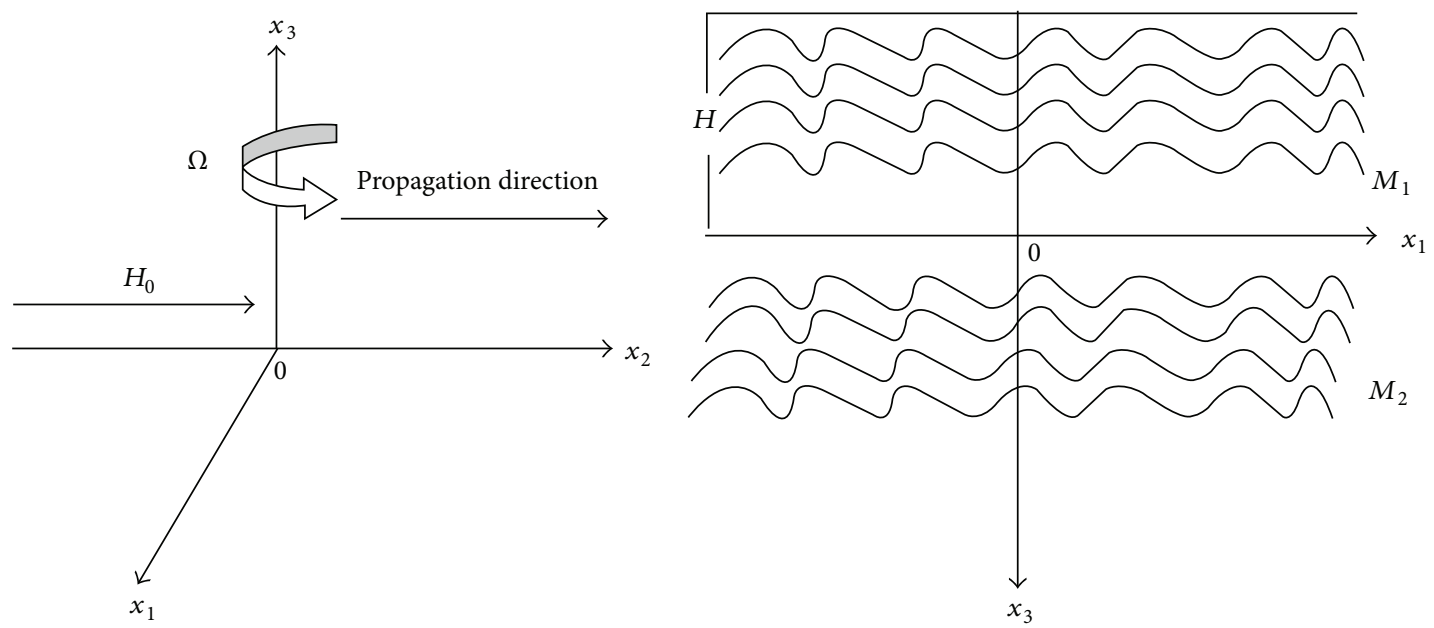

FIgURE 1: Schematic of the problem.

where we have used

$$
\begin{gathered}
\vec{H}=\overrightarrow{H_{0}}+\vec{h}(x, z, t), \quad \overrightarrow{H_{0}}=(0, H, 0) \\
\vec{B}=\mu_{e} \vec{H}=\mu_{e} H \vec{J} ;
\end{gathered}
$$

then

$$
\begin{aligned}
F_{x} & =B J_{z}=\left[\mu_{e} H\right] H\left(\frac{\partial^{2} u}{\partial x_{1}^{2}}+\frac{\partial^{2} w}{\partial x_{1} \partial x_{3}}\right) \\
& =\mu_{e} H^{2}\left(\frac{\partial^{2} u}{\partial x_{1}^{2}}+\frac{\partial^{2} w}{\partial x_{1} \partial x_{3}}\right), \\
F_{z} & =B J_{x}=\left[\mu_{e} H\right] H\left(\frac{\partial^{2} u}{\partial x_{3} \partial x_{1}}+\frac{\partial^{2} w}{\partial x_{3}^{2}}\right) \\
& =\mu_{e} H^{2}\left(\frac{\partial^{2} u}{\partial x_{3} \partial x_{1}}+\frac{\partial^{2} w}{\partial x_{3}^{2}}\right) .
\end{aligned}
$$

(iii) Equation of heat conduction is

$$
\rho c_{E}\left(\dot{T}+\tau_{0} \ddot{T}\right)+\beta_{1} T_{0} \dot{\varepsilon}_{k k}+a T_{0}\left(\dot{C}+\tau_{0} \ddot{C}\right)=K T_{, i i} .
$$

(iv) Equation of mass diffusion is

$$
D \beta_{2} \varepsilon_{k k, i i}+D a\left(T+\tau_{1} \frac{\partial T}{\partial t}\right)_{, i i}+\dot{C}-D b\left(C+\tau^{\prime} \frac{\partial C}{\partial t}\right)_{, i i}=0 .
$$

Here, the medium is rotating with angular velocity $\Omega=$ $\Omega \underline{n}$, where $\underline{n}$ is the unit vector along the axis of rotation and this equation of motion includes two additional terms, namely,

(i) the centripetal acceleration $\vec{\Omega} \times \vec{\Omega} \times \vec{u}$ due to timevarying motion,

(ii) the Carioles acceleration $2 \vec{\Omega} \times \dot{\vec{u}}$, where $\beta_{1}=(3 \lambda+2 \mu) \alpha_{t}$ and $\beta_{2}=(3 \lambda+2 \mu) \alpha_{c}, \lambda$ and $\mu$ are Lame's constants, $\alpha_{t}$ is the coefficient of linear thermal expansion, $\rho$ and $C_{E}$ are, respectively, the density and specific heat at constant strain, $a, b$ are, respectively, coefficient describing the measure of thermoelastic diffusion effects and of diffusion effects, $T_{0}$ is the reference temperature assumed to be such that $\left|T / T_{0}\right| \ll 1, \tau_{0}, \tau_{1}$ are thermal relaxation times with $\tau_{1} \geq \tau_{0}>0$ and $\tau^{0}, \tau^{\prime}$ are diffusion relaxation times with $\tau^{\prime} \geq \tau^{0}>0$, and $u_{i}$ are components of displacement vector. $T(x, y, z)$ is the temperature change and $C$ is the concentration; $\sigma_{i j}\left(=\sigma_{j i}\right), \varepsilon_{i j}=\left(u_{i, j}+u_{j, i}\right) / 2$ are, respectively, the components of stress and strain tensor.

The symbols correspond to partial derivative and time derivative, respectively.

Following Bullen [33], the equations of motion and constitutive relations in isotropic elastic medium are given by

$$
\begin{gathered}
\left(\lambda^{e}+\mu^{e}\right) u_{j, i j}^{e}+\mu^{e} u_{i, j j}^{e}+F_{i}^{e}=\rho^{e} \ddot{u}_{i}^{e}, \\
\sigma_{j i}^{e}=\lambda^{e} \theta^{e} \delta_{i j}+2 \mu^{e} \varepsilon_{i j}^{e}, \quad i=j=1,2,3,
\end{gathered}
$$

where

$$
\Theta^{e}=u_{k, k}^{e}
$$

$$
\varepsilon_{i j}^{e}=\frac{u_{i, j}^{e}+u_{j, i}^{e}}{2}, \quad i=j=1,2,3
$$

and $u^{e}=\left(u_{1}^{e}, u_{2}^{e}, u_{3}^{e}\right)$ is the displacement vector, $\rho^{e}$ is the density of the isotropic medium and $\lambda^{e}, \mu^{e}$ are Lame's constants, $\sigma_{j i}^{e}\left(=\sigma_{i j}^{e}\right)$ are components of stress tensor, and $\delta_{i j}$ is the Kronecker delta.

\section{Formulation of the Problem}

As shown in Figure 1, we consider an isotropic elastic layer (Medium $M_{1}$ ) of thickness $H$ overlaying a homogeneous, isotropic, generalized thermodiffusive elastic half-space in rotating frame of reference (Medium $M_{2}$ ). The origin of the coordinate system $(x, y, z)$ is taken at any point on 
the horizontal surface and $x_{2}$-axis in the direction of wave propagation and $x_{3}$-axis taking vertically downward into half-space, so that all particles on a line parallel to $x_{2}$-axis are equally displaced. Therefore, all the field quantities will be independent of $x_{2}$-axis coordinate. The interface between isotropic elastic layer and thermodiffusive elastic half-space with rotation has been taken at an imperfect boundary. The displacement vector $u$, temperature $T$, concentration $C$, and rotation for medium $M_{2}$ are taken as

$$
\begin{gathered}
\vec{u}=\left(u_{1}, 0, u_{3}\right), T\left(x_{1}, x_{3}, t\right), C\left(x_{1}, x_{3}, t\right), \\
\Omega=(0, \Omega, 0)
\end{gathered}
$$

and displacement vector $\vec{u}^{e}$ for the layer (Medium $M_{1}$ ) is taken as

$$
\vec{u}^{e}=\left(u_{1}^{e}, 0, u_{3}^{e}\right)
$$

We define the dimensionless quantities

$$
\begin{gathered}
x_{i}^{\prime}=\frac{\omega_{1}^{*} x_{i}}{v_{1}}, \quad u_{i}^{\prime}=\frac{\omega_{1}^{*} u_{i}}{v_{1}}, \quad t^{\prime}=\omega_{1}^{*} t, \quad \tau_{1}^{\prime}=\omega_{1}^{*} \tau, \\
T^{\prime}=\frac{\beta_{1} T}{\rho v_{1}^{2}}, \quad C^{\prime}=\frac{\beta_{2} C}{\rho v_{1}^{2}}, \quad \tau_{0}^{\prime}=\omega_{1}^{*} \tau_{0}, \quad \tau^{0 \prime}=\omega_{1}^{*} \tau^{0}, \\
\tau^{\prime \prime}=\omega_{1}^{*} \tau^{\prime}, \quad \sigma_{i j}^{\prime}=\frac{\sigma_{i j}}{a_{1} T_{0}}, \quad \Omega^{\prime}=\frac{\Omega}{\omega_{1}^{*}}, \\
K_{n}^{\prime}=\frac{v_{1} k_{n}}{\beta_{1} T_{0} \omega_{1}^{*}}, \quad K_{1}^{\prime}=\frac{v_{1} k_{1}}{\beta_{1} T_{0} \omega_{1}^{*}}, \\
v_{1}^{2}=\frac{\lambda+2 \mu+\mu_{e} H^{2}}{\rho}, \quad \omega_{1}^{*}=\frac{\rho c_{E} v_{1}^{2}}{k_{1}} .
\end{gathered}
$$

Upon introducing the quantities in (1)-(3), (8), and (14)(16), after suppressing the primes, with the aid of (17) and (18), we obtain

$$
\begin{aligned}
& u_{1,11}+ \delta_{2} u_{3,13}+\delta_{1} u_{1,33}-\tau_{\Theta} T_{.1}-\tau_{c}^{\prime} C_{, 1} \\
&= {\left[\ddot{u}_{1}-\Omega^{2} u_{1}+2 \Omega \dot{u}_{3}\right], } \\
& \delta_{1} u_{3,11}+\delta_{2} u_{1.31}+u_{3,33}-\tau_{\theta} T_{.3}-\tau_{c}^{\prime} C_{, 3} \\
&=\left[\ddot{u}_{3}-\Omega^{2} u_{3}-2 \Omega \dot{u}_{1}\right] \\
& \nabla^{2} T=\tau_{\theta}^{0} \dot{T}+\chi_{1} \tau_{f}^{0} \dot{C}+\chi_{2} \dot{e} \\
& q_{1}^{*} \nabla^{2} e+q_{2}^{*} \tau_{\theta}^{\prime} \nabla^{2} T-q_{3}^{*} \tau_{c}^{\prime} \nabla^{2}+\dot{C}=0, \\
& \frac{\left(u_{1,11}^{e}+u_{3,13}^{e}\right)}{\delta_{3}^{2}}+\frac{\nabla^{2} u_{1}^{e}}{\delta_{4}^{2}}=\ddot{u}_{1}^{e}, \\
& \frac{\left(u_{1,13}^{e}+u_{3,33}^{e}\right)}{\delta_{3}^{2}}+\frac{\nabla^{2} u_{3}^{e}}{\delta_{4}^{2}}=\ddot{u}_{3}^{e},
\end{aligned}
$$

where

$$
\begin{aligned}
& \delta_{1}=\frac{\mu}{\left(\lambda+2 \mu+\mu^{e} H\right)}, \quad \delta_{2}=\frac{\lambda+\mu+\mu^{e} H}{\left(\lambda+2 \mu+\mu^{e} H\right)}, \\
& \chi_{1}=\frac{a T_{0} v_{1}^{2} \beta_{1}}{\omega_{1}^{*} k \beta_{2}}, \quad \chi_{2}=\frac{T_{0} \beta_{1}^{2}}{\rho k \omega_{1}^{*}}, \\
& q_{1}^{*}=\frac{D \omega_{1}^{*} \beta_{2}^{2}}{\rho v_{1}^{2}}, \quad q_{2}^{*}=\frac{D \omega_{1}^{*} b}{v_{1}^{2}}, \\
& q_{3}^{*}=\frac{D \omega_{1}^{*} \beta_{2} a}{\beta_{1} v_{1}^{2}}, \quad \nabla^{2}=\frac{\partial^{2}}{\partial x^{2}}+\frac{\partial^{2}}{\partial Z^{2}}, \\
& e=\frac{\partial u}{\partial x}+\frac{\partial w}{\partial z}, \quad \delta_{4}^{2}=\frac{\rho^{e} v_{1}^{2}}{\mu^{e}} \\
& \delta_{3}^{2}=\frac{\rho^{e} v_{1}^{2}}{\mu_{e} H^{2}+\lambda^{e}+\mu^{e}}, \quad \delta_{5}^{2}=\frac{\rho^{e} v_{1}^{2}}{\lambda^{e}+2 \mu^{e} \mu_{e} H^{2}}, \\
& \tau_{\theta}^{\prime}=1+\tau_{1} \frac{\partial}{\partial t}, \quad \tau_{\theta}^{0}=1+\tau_{0} \frac{\partial}{\partial t}, \\
& \tau_{c}^{\prime}=1+\tau^{\prime} \frac{\partial}{\partial t}, \quad \tau_{f}^{0}=1+\tau^{0} \frac{\partial}{\partial t} \\
& R_{H}^{2}=\frac{\mu^{e} H}{\lambda+2 \mu} \text {. }
\end{aligned}
$$

For an isotropic elastic layer, we introduce potential functions $\Phi$ and $\Psi$ through the relations

$$
u_{1}^{e}=\frac{\partial \Phi}{\partial x_{1}}-\frac{\partial \Psi}{\partial x_{3}}, \quad u_{3}^{e}=\frac{\partial \Phi}{\partial x_{3}}+\frac{\partial \Psi}{\partial x_{1}}
$$

Substituting from (22) into (20), we have

$$
\begin{aligned}
& \Phi_{, 11}+\Phi_{, 33}-\delta_{5}^{2} \ddot{\Phi}=0, \\
& \Psi_{, 11}+\Psi_{, 33}-\delta_{4}^{2} \ddot{\Psi}=0 .
\end{aligned}
$$

\section{Solution of the Problem}

To solve (19), (22), and (23), we assume the solution in the form

$$
\begin{aligned}
& \left(u_{1}, u_{2}, T, C, \Phi, \Psi\right) \\
& \quad=\left(1, W, S, R, P_{1} P_{2}\right) U \exp \left[i \xi\left(x_{1}+m x_{3}-c t\right)\right],
\end{aligned}
$$

where $c=\omega / \xi$ is the nondimensional phase velocity, $\omega$ is the frequency, $m$ is still parameter 1 , and $W, S$, and $R$ are, respectively, the amplitude ratio of $u_{1}, u_{3}, T$, and $C$ with 
respect to $u_{1}$. Substituting the values of $u_{1}, u_{3}, T$, and $C$ from (25) in (19), we obtain

$$
\begin{aligned}
& 1+\delta_{1} m^{2}-c^{2}\left(1+\Lambda^{2}\right)+\left(\delta_{2} m-2 i \Lambda c^{2}\right) W \\
&+i c \omega^{-1}\left(\tau_{11} S+\tau_{21} R\right)=0, \\
&\left(\delta_{2} m\right.\left.-2 \Lambda i c^{2}\right)+\left(\delta_{1}+m^{2}-c^{2}\left(1+\Lambda^{2}\right)\right) W \\
&+i c \omega^{-1} m\left(\tau_{11} S+\tau_{21} R\right)=0, \\
& \chi_{2} C+ \chi_{2} C m W-i \omega^{-1} c^{2} \chi_{1} \tau_{20} R \\
&-i \omega^{-1}\left(i \omega\left(1+m^{2}\right)+c^{2} \tau_{10}\right) S=0, \\
& q_{1}^{*}\left(1+m^{2}\right)+q_{1}^{*}\left(1+m^{2}\right) m w-i q_{2}^{*}\left(1+m^{2}\right) \tau_{11} c \omega^{-1} S \\
& \quad+\left[i q_{3}^{*}\left(1+m^{2}\right) \tau_{21} c \omega^{-1}+c^{3} \omega^{-2}\right] R=0,
\end{aligned}
$$

where

$$
\begin{gathered}
\Lambda=\Omega \omega^{-1}, \quad \tau_{11}=1-i \omega \tau_{1}, \quad \tau_{21}=1-i \omega \tau^{\prime}, \\
\tau_{10}=1-i \omega \tau_{0}, \quad \tau_{20}=1-i \omega \tau^{0} .
\end{gathered}
$$

The system of (26) has a nontrivial solution if the determinant of the coefficient $[1, W, S, R]^{T}$ vanishes, which yield to the following polynomial characteristic equation:

$$
m^{8}+A^{*} m^{6}+B^{*} m^{4}+C^{*} m^{2}+D^{*}=0 .
$$

The constants $A^{*}, B^{*}, C^{*}$, and $D^{*}$ are given in Appendix A. The characteristic equation (28) is biquadratic in $m^{2}$ and hence possesses four roots, $m_{p}^{2} ; p=1,2,3,4$ corresponding to four roots; there exist three types of quasilongitudinal waves and one quasitransverse wave. The formal expression for displacement, temperature, and concentration satisfying the radiation condition $\operatorname{Re}\left(m_{p}\right) \geq 0$ can be written as

$$
\left(u_{1}, u_{3}, T, C\right)=\sum_{P=1}^{4}\left(1, n_{1 p}, n_{2 p}, n_{3 p}\right) \exp \left[i \xi\left(x+i m_{p} z-c t\right)\right] .
$$

Substituting the values of $\Phi$ and $\Psi$ from (25) in (23) and (24) and with the aid of (22), we obtain

$$
\begin{aligned}
& u_{1}^{e}=i \xi\left(B_{1} c_{5}+B_{2} s_{5}\right)+\xi m_{6}\left(D_{1} s_{6}-D_{2} c_{6}\right) \exp i \xi(x-c t), \\
& u_{3}^{e}=\xi m_{5}\left(B_{2} c_{5}-B_{1} s_{5}\right)+i \xi\left(D_{1} c_{6}+D_{2} s_{6}\right) \exp i \xi(x-c t),
\end{aligned}
$$

where

$$
\begin{array}{ll}
m_{5}=\sqrt{c^{2} \delta_{5}^{2}-1}, & m_{6}=\sqrt{c^{2} \delta_{4}^{2}-1}, \\
c_{5}=\cos \left(\xi m_{5} x_{3}\right), & c_{6}=\cos \left(\xi m_{5} x_{3}\right), \\
s_{5}=\sin \left(\xi m_{5} x_{3}\right), & s_{6}=\sin \left(\xi m_{6} x_{3}\right),
\end{array}
$$

where $A_{P}(P=1,2,3,4), B_{1}, B_{2}, D_{1}, D_{2}$ are arbitrary constants. The coupling constants $n_{1 p}, n_{2 p}, n_{3 p}(p=1,2,3,4)$ are given in Appendix B.

\section{Boundary Conditions}

In this paper, linear model is adopted to represent the imperfectly bonded interface conditions. The boundary conditions are the vanishing of the normal stress; Maxwell's electromagnetic stress tensor $\tau_{i j}$ is given by

$$
\tau_{i j}=\mu_{e}\left[H_{i} h_{j}+H_{j} h_{i}-H_{k} h_{k} \delta_{i j}\right]
$$

and tangential stress at free surface. The discontinuities in displacements have linear relations with stresses, continuity of normal, Maxwell's electromagnetic stress tensor and tangential stress, vanishing of the gradient of temperature, and concentration at the interface between the isotropic elastic layer and isotropic thermodiffusive elastic half-space. Mathematically, these can be written as follows.

(i) Mechanical conditions:

$$
\begin{gathered}
\left(\sigma_{33}^{e}\right)_{M_{1}}+\tau_{33}^{e}=0 \quad\left(\sigma_{31}^{e}\right)_{M_{1}}+\tau_{31}^{e}=0 \\
x_{3}=-H, \\
\left(\sigma_{33}^{e}\right)_{M_{1}}+\tau_{33}^{e}=\left(\sigma_{33}\right)_{M_{2}}+\tau_{33} \\
\left(\sigma_{31}^{e}\right)_{M_{1}}+\tau_{31}^{e}=\left(\sigma_{31}\right)_{M_{2}}+\tau_{31} \\
\left(\sigma_{33}\right)_{M_{1}}+\tau_{33}^{e}=k_{n}\left[\left(u_{3}\right)_{M_{2}}-\left(u_{3}\right)_{M_{1}}\right] \\
\left(\sigma_{31}\right)_{M_{1}}+\tau_{31}^{e}=k_{t}\left[\left(u_{3}\right)_{M_{2}}-\left(u_{3}\right)_{M_{1}}\right] \\
x_{3}=0
\end{gathered}
$$

(ii) Temperature condition

$$
\frac{\partial T}{\partial x_{3}}=0, \quad x_{3}=0
$$

(iii) Concentration condition

$$
\frac{\partial C}{\partial x_{3}}=0, \quad x_{3}=0
$$

where $k_{n}$ and $k_{t}$ are the normal and transverse stiffness of layer which have dimension $\mathrm{Nm}^{-3}$.

\section{Derivation of the Secular Equations}

Substituting the value of $u_{1}, u_{3}, T, C, u_{1}^{e}, u_{3}^{e}$ from (29)-(30) into (33)-(35), with the aid of (1), (10) and (13)-(14), after simplification we obtain

$$
b_{5} \tan \left(\xi m_{5} H\right) \Delta_{1}+b_{5} \Delta_{2}+\frac{b_{6} s_{6}}{c_{5}} \Delta_{3}-\frac{b_{6} c_{6}}{c_{5}} \Delta_{4}=0,
$$

where $\Delta_{1}=\left|R_{i j}\right|_{7 \times 7}$ the entries $R_{i j}$ of the determinant are given in Appendix $\mathrm{C}$ and $\Delta_{2}$ is obtained by replacing the first column of $\Delta_{1}$ by $\left[\begin{array}{lllllll}R_{11}^{*} & 0 & 0 & R_{41}^{*} & R_{51}^{*} & R_{61}^{*} & R_{71}^{*}\end{array}\right]^{T}, \Delta_{3}$ is obtained by replacing the second column of $\Delta_{2}$ by $\left[\begin{array}{lll}R_{12}^{*} & 0 & 0\end{array}\right.$ $\left.\begin{array}{llll}R_{42}^{*} & R_{52}^{*} & R_{62}^{*} & R_{72}^{*}\end{array}\right]^{T}$, and $\Delta_{4}$ is obtained by replacing 
the third column of $\Delta_{3}$ by $\left[\begin{array}{lllllll}R_{13}^{*} & 0 & 0 & R_{43}^{*} & R_{53}^{*} & R_{63}^{*} & R_{73}^{*}\end{array}\right]^{T}$. The entries of $\Delta_{P}(P=1,2,3,4)$ are given in Appendix C.

If we write

$$
c^{-1}=v^{-1}+i \omega^{-1} G
$$

then $\xi=F+i G$ where $F=\omega / v$ and $G$ are real numbers. Also the roots of characteristic equations are in general complex.

Hence, assume that $m_{p}=p_{p}+i q_{p}$ so that exponent in the plane wave solutions in (25) becomes

$$
i F\left(x_{1}-m_{p}^{A} x_{3}-v t\right)-F\left(\frac{G}{F} x_{1}+m_{p}^{R} x_{3}\right),
$$

where

$$
m_{p}^{A}=\frac{G}{F} p_{p}+q_{p}, \quad m_{p}^{R}=p_{p}-i \frac{G}{F} q_{p} .
$$

This shows that $v$ is the propagation velocity and $G$ is the attenuation coefficient of the wave. Upon using the representation (37) in secular equation (36), the values of propagation speed $v$ and attenuation coefficient $G$ of wave propagation can be obtained.

\section{Particular Cases}

(i) Normal Stiffness. In this case, $k_{n} \neq 0, k_{t} \rightarrow \infty$, and the secular equation (36) remains the same. But the following will be replaced in the values of $\Delta_{p}(p=1,2,3,4)$ :

$$
\begin{gathered}
R_{71}=\frac{-i \xi}{\beta_{1} T_{0}}, \quad R_{72}=0, \quad R_{73}=\frac{\xi m_{6}}{\beta_{1} T_{0}}, \\
R_{74}=R_{75}=R_{76}=R_{77}=1, \\
R_{71}^{*}=0, \quad R_{72}^{*}=-i \xi, \quad R_{73}^{*}=0 .
\end{gathered}
$$

(ii) Tangential Stiffness. In this case $k_{t} \neq 0, k_{n} \rightarrow \infty$, and the secular equation (36) remains the same with the change of values of $\Delta_{p}(p=1,2,3,4)$ by taking

$$
\begin{gathered}
R_{61}=-\frac{i \xi}{\beta_{1} T_{0}}, \quad R_{62}=0, \quad R_{63}=\xi m_{6} \frac{1}{\beta_{1} T_{0}}, \\
R_{64}=n_{12}, \quad R_{65}=n_{11}, \quad R_{66}=n_{13}, \quad R_{67}=n_{14}, \\
R_{61}^{*}=0, \quad R_{62}^{*}=-\xi m_{5} \frac{1}{\beta_{1} T_{0}}, \quad R_{63}^{*}=-i \xi \frac{1}{\beta_{1} T_{0}} .
\end{gathered}
$$

(iii) Welded Contact. In this case, $k_{n} \rightarrow \infty, k_{t} \rightarrow \infty$, and the secular equation (36) remains the same, but the value of $\Delta_{p}(p=1,2,3,4)$ is given by replacing

$$
\begin{gathered}
R_{61}=-i \xi \frac{1}{\beta_{1} T_{0}}, \quad R_{62}=0, \quad R_{63}=\xi m_{6} \frac{1}{\beta_{1} T_{0}}, \\
R_{64}=n_{12}, \quad R_{65}=n_{11}, \quad R_{66}=n_{13}, \quad R_{67}=n_{14},
\end{gathered}
$$

$$
\begin{gathered}
R_{61}^{*}=0, \quad R_{72}^{*}=-\xi m_{5} \frac{1}{\beta_{1} T_{0}}, \quad R_{63}^{*}=-i \xi \frac{1}{\beta_{1} T_{0}}, \\
R_{61}=-i \xi \frac{1}{\beta_{1} T_{0}}, \quad R_{62}=0, \quad R_{63}=\xi m_{6} \frac{1}{\beta_{1} T_{0}} \\
R_{64}=n_{12}, \quad R_{65}=n_{11}, \quad R_{66}=n_{13}, \quad R_{67}=n_{14}, \\
R_{61}^{*}=0, \quad R_{72}^{*}=-\xi m_{5} \frac{1}{\beta_{1} T_{0}}, \quad R_{63}^{*}=-i \xi \frac{1}{\beta_{1} T_{0}} .
\end{gathered}
$$

\section{Special Cases}

Case (i). If we take $\Omega=0$, that is, in the absence of rotation effect, the frequency equation (36) will reduce to the frequency equation for an isotropic elastic layer and a homogenous isotropic thermodiffusive elastic half-space without rotation.

Case (ii). If we take $H=0, \Omega=0, \tau^{\prime}>0$, and $\tau_{1}>0$ and in the absence of diffusion effect, that is, $b_{1}=b_{3}=a=b=0$, (36) will reduce to the frequency equation for Rayleigh wave

$$
\begin{aligned}
& \left(2-\frac{c^{2}}{\delta_{1}^{2}}\right)^{2}\left(\bar{m}_{1}^{2}+\bar{m}_{2}^{2}+\bar{m}_{1} \bar{m}_{2}-1+c^{2}\right) \\
& -4 \bar{m}_{1} \bar{m}_{2} \bar{m}_{3}\left(\bar{m}_{1}+\bar{m}_{2}\right)=0 .
\end{aligned}
$$

Here, $m_{3}=\sqrt{1-c^{2} / \delta_{1}^{2}}$ and $\bar{m}_{p}(p=1,2)$ are the roots of (28), obtained by taking $b_{1}=b_{3}=a=b=0$ and $\tau^{\prime}>0$, $\tau_{1}>0$. The resulting equation (43) is similar to (22) as given by Dawn and Chakraborty [26].

Case (iii). In the absence of isotropic elastic layer and thermal and diffusion effects, we obtain the frequency equation corresponding to isotropic elastic half-space by changing the dimensionless quantities into physical quantities as

$$
\left(2-\frac{c^{2}}{c_{2}^{2}}\right)^{2}=4\left(1-\frac{c^{2}}{c_{1}^{2}}\right)^{1 / 2}\left(1-\frac{c^{2}}{c_{2}^{2}}\right)^{1 / 2}
$$

where $c_{1}^{2}=\left(\lambda+\mu+\mu^{e}\right) / \rho, c_{2}^{2}=\mu / \rho$.

The frequency equation (36) is the same as derived in Ewing et al. [34].

\section{Surface Displacements, Temperature Change, and Concentration}

The amplitude of surface displacements, temperature change, and concentration at the surface $z=0$ during Rayleigh wave propagation in the cases of stress free, vanishing of 
the gradient of temperature, and concentration of the halfspace are

$$
\begin{aligned}
& u_{1}=\bar{E} A \exp \left[i F\left(x_{1}-v t\right)\right], \\
& u_{3}=\bar{R} A \exp \left[i F\left(x_{1}-v t\right)\right], \\
& T=\bar{W} A \exp \left[i F\left(x_{1}-v t\right)\right], \\
& C=\bar{L} A \exp \left[i F\left(x_{1}-v t\right)\right],
\end{aligned}
$$

where

$$
\begin{gathered}
A=A_{1} \exp \left(-F x_{1}\right), \\
\bar{E}=\frac{F_{1}^{*}-F_{2}^{*}+F_{3}^{*}-F_{4}^{*}}{F_{1}^{*}}, \\
\bar{R}=\frac{\left(n_{11} F_{1}^{*}-n_{12} F_{2}^{*}+n_{13} F_{3}^{*}-n_{14} F_{4}^{*}\right)}{F_{1}^{*}}, \\
\bar{W}=\frac{\left(n_{21} F_{1}^{*}-n_{22} F_{2}^{*}+n_{23} F_{3}^{*}-n_{24} F_{4}^{*}\right)}{F_{1}^{*}}, \\
\bar{L}=\frac{\left(n_{31} F_{1}^{*}-n_{32} F_{2}^{*}+n_{33} F_{3}^{*}-n_{34} F_{4}^{*}\right)}{F_{1}^{*}}
\end{gathered}
$$

and $F_{P}^{*}(P=1,2,3,4)$ are given in Appendix D.

\section{Specific Loss}

The specific loss is the ratio of energy $(\Delta w)$ dissipated in taking a specimen through a stress cycle, to the elastic energy $(w)$ stored in the specimen when the strain is maximum. The specific loss is the most direct method of defining internal friction for a material. For a sinusoidal plane wave of small amplitude, Kolsky [35] shows that the specific loss $\Delta w / w$ equals $4 \pi$ times the absolute value of imaginary part of $\xi$ to the real part of $\xi$; that is,

$$
\begin{aligned}
\frac{\Delta \omega}{w} & =4 \pi\left|\frac{\operatorname{Im}(\xi)}{\operatorname{Re}(\xi)}\right|=4 \pi\left|\frac{i G}{F}\right|=4 \pi\left|\frac{i G}{\omega / v}\right| \\
& =4 \pi\left|\frac{i v G}{\omega}\right|=4 \pi\left|\frac{1}{F}(i G)\right| .
\end{aligned}
$$

\section{Numerical Results and Discussion}

Following Sherief and Saleh [16], we take the following values of relevant parameter for the copper material:

$$
\begin{gathered}
\lambda=7.76 \times 10^{10} \mathrm{Kgm}^{-1} \mathrm{~s}^{-2}, \quad \mu=3.86 \times 10^{10} \mathrm{Kgm}^{-1} \mathrm{~s}^{-2}, \\
T_{0}=0.293 \times 10^{3} \mathrm{~K} \quad \rho=8.954 \times 10^{3} \mathrm{kgm}^{-3}, \\
a=1.2 \times 10^{4} \mathrm{~m}^{2} \mathrm{~s}^{-2} \mathrm{k}^{-1}, \quad b=9 \times 10^{5} \mathrm{~m}^{5} \mathrm{~s}^{-2} \mathrm{gk}^{-1}
\end{gathered}
$$

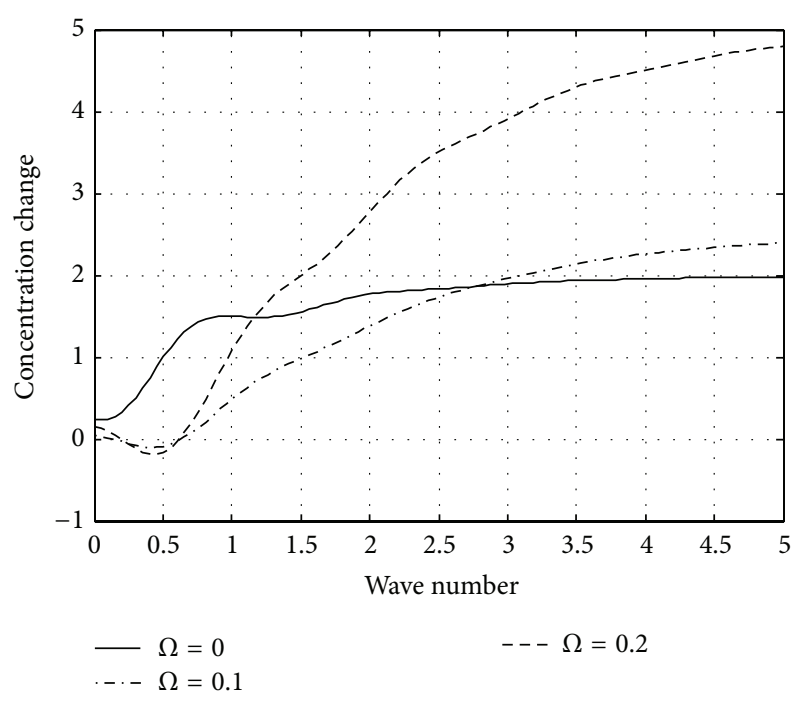

FIGURE 2: Variation of concentration with respect to the wave number with variation of rotation.

$$
\begin{gathered}
c_{E}=3831 \times 10^{3} \mathrm{JKg}^{-1} \mathrm{~K}^{-1}, \quad \alpha_{t}=1.78 \times 10^{-5} \mathrm{k}^{-1}, \\
\alpha_{c}=1.98 \times 10^{-4} \mathrm{k}^{-1} \mathrm{~m}^{3} \mathrm{~g} \quad K=0.383 \times 10^{3} \mathrm{Wm}^{-1} \mathrm{k}^{-1}, \\
D=0.85 \times 10^{-8} \mathrm{Sm}^{-3} \mathrm{~g}^{-1}, \quad \tau_{0}=0.07 \mathrm{~s}, \\
\tau^{1}=0.03, \quad \tau^{0}=0.04, \quad \tau_{1}=0.07 \mathrm{~s} .
\end{gathered}
$$

The elastic constants for granite are given by Bullen [33]:

$$
\begin{gathered}
\lambda^{e}=\mu^{e}=2.238 \times 10^{3} \mathrm{~J} \cdot \mathrm{Kgm}^{-1} \mathrm{~K}^{-1}, \\
\rho^{e}=2.65 \times 10^{3} \mathrm{~J} \cdot \mathrm{kgm}^{-3} .
\end{gathered}
$$

The concentration change, phase velocity and attenuation coefficient of wave propagation, displacement, stresses, temperature in the context of Green Lindsay (GL) theory of thermoelastic diffusion with variations of magnetic field, and rotation in $2 \mathrm{D}$ and $3 \mathrm{D}$ have been computed for various values of nondimensional wave number and calculated numerically and represented graphically in Figures 2-22.

Figure 2 displays the variation of concentration change with different values of rotation with respect to the wave number; it appears that the concentration change increases with an increasing of the wave number and if there is no rotation and small wave number takes larger values than in the presence of rotation and large values of the wave number. Figures 3 and 4 clear the phase velocity and attenuation coefficients variations with respect to the wave number with variation of the rotation; it is concluded that the phase velocity begins from 1.2 but the attenuation coefficient begins from zero if the wave number equals zero and tends to zero if the wave number tends to infinity; also, it is seen that they increase and decrease periodically with an increasing of the wave number and rotation that indicate the interruption of the phase velocity and attenuation coefficient with the largest values of the wave number and rotation that agree 


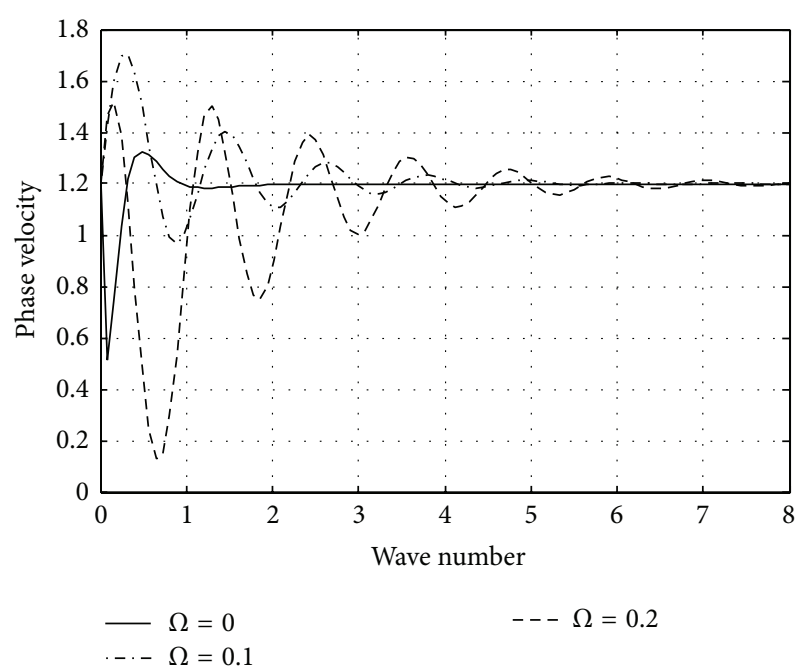

FIGURE 3: Variation of phase velocity with respect to the wave number with variation of rotation.

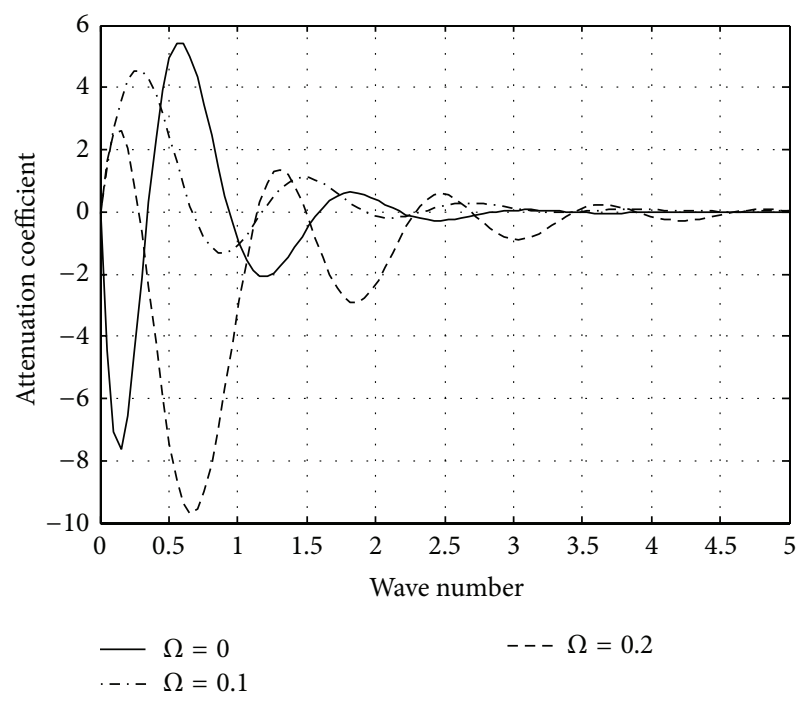

FIGURE 4: Variation of attenuation coefficient with respect to the wave number with variation of rotation.

with the practical results. Figure 5 shows the variation of the displacement component with respect to the wave number with variation of the rotation; it is concluded that it takes various values for zeros value of the wave number and tends to zero if the wave number tends to infinity; also, it seems that it increases and decreases periodically with an increasing of the wave number and rotation.

Figures 6 and 7 make clear the variation of the stresses components with respect to the wave number with variation of the rotation; it appears that it begins from zero for zeros value of the wave number and tends to zero if the wave number tends to infinity; also, it is shown that it increases and decreases periodically with an increasing of the wave number and rotation. Figure 8 displays the variation of the temperature with various values of the wave number and rotation; one can see that it begins from zero for zeros wave

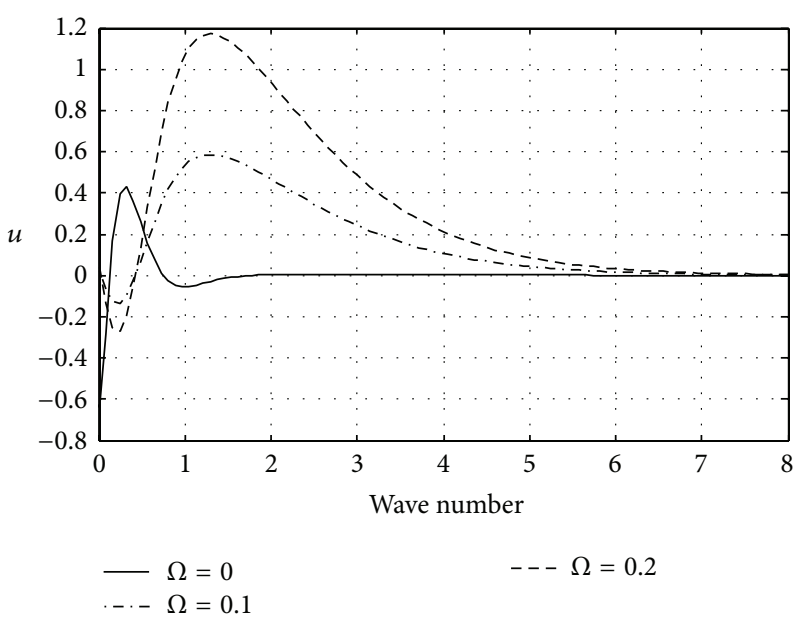

FIgURE 5: Variation of the displacement $u$ with respect to the wave number with variation of rotation.

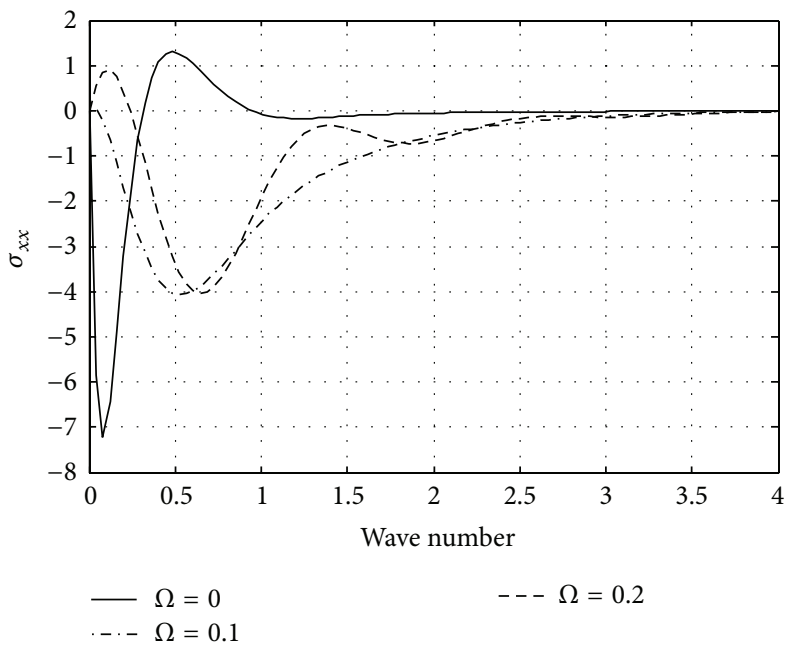

FIGURE 6: Variation of normal stress $\sigma_{x x}$ with respect to the wave number with variation of rotation.

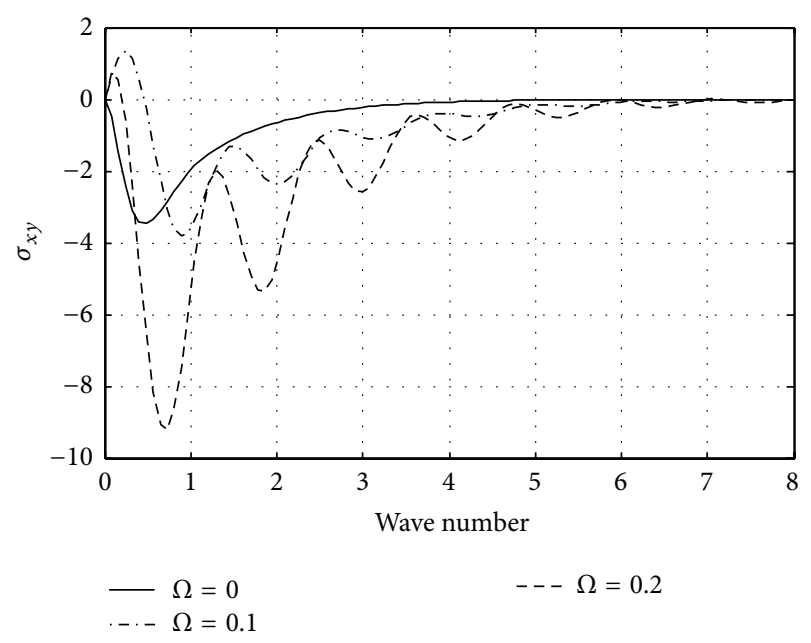

FIGURE 7: Variation of shear stress $\sigma_{x y}$ with respect to the wave number with variation of rotation. 


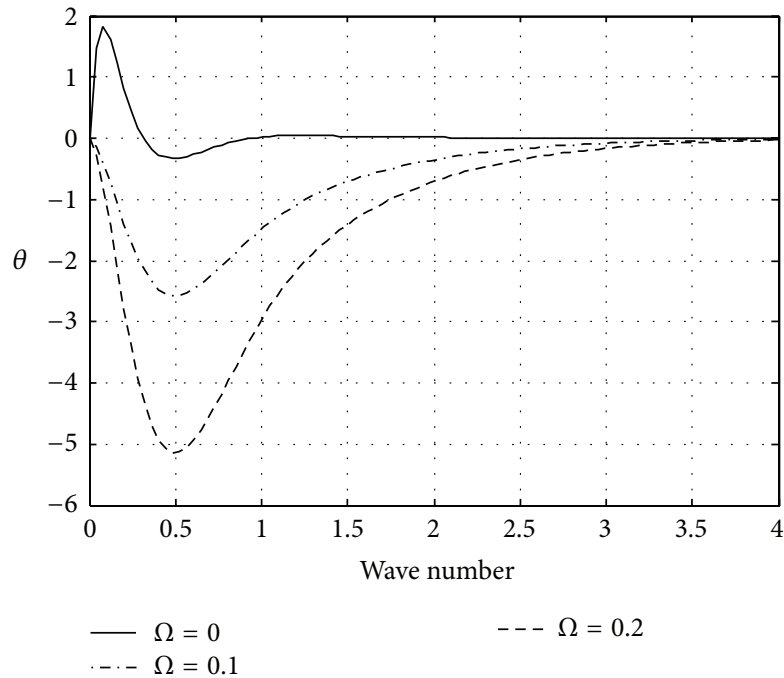

FIGURE 8: Variation of the temperature $\theta$ with respect to the wave number with variation of rotation.

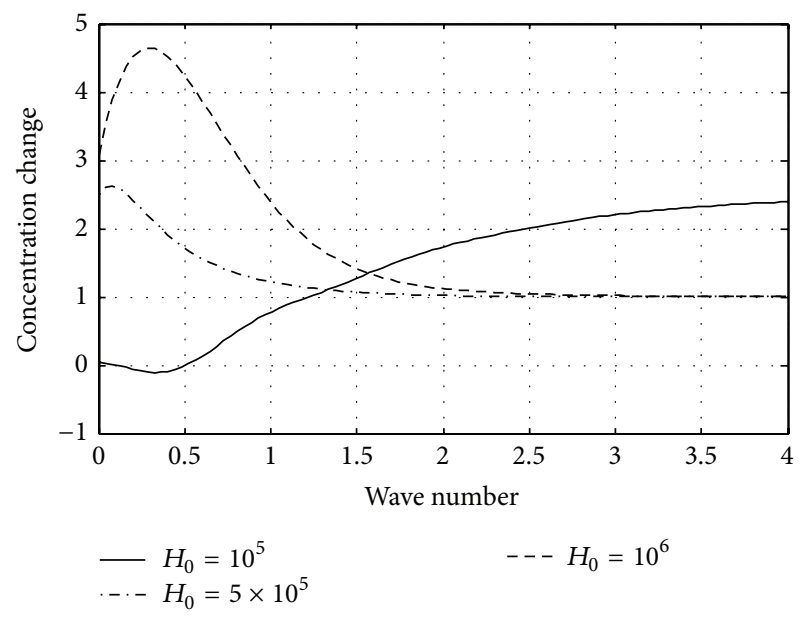

FIGURE 9: Variation of the concentration charge with respect to the wave number with variation of the magnetic field.

number and tends to zero, decreases and increases tends to zero if the wave number tends to infinity; it is clear that the temperature decreases with increasing rotation values that physically indicates the negative influence of rotation on the temperature that takes into consideration engineering and structures. Also, it is seen that, in the absence of rotation, all values of the concentration change, phase velocity, attenuation coefficient, displacement, stresses components, and temperature take a smooth behavior comparing with the corresponding values in the presence of rotation that take harmonic behavior.

Figure 9 shows the variation of concentration change with various values of magnetic field with respect to the wave number; it appears that the concentration change increases with an increasing of the wave number and decreases and arrives to the unity if the wave number tends to infinity; if the magnetic field is absent, the concentration change smallest

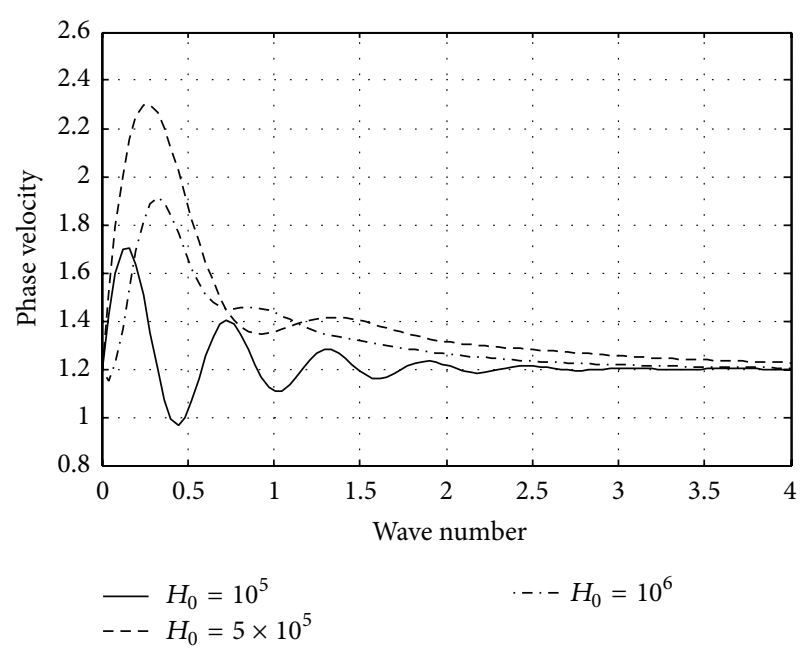

FIGURE 10: Variation of phase velocity with respect to the wave number with variation of the magnetic field.

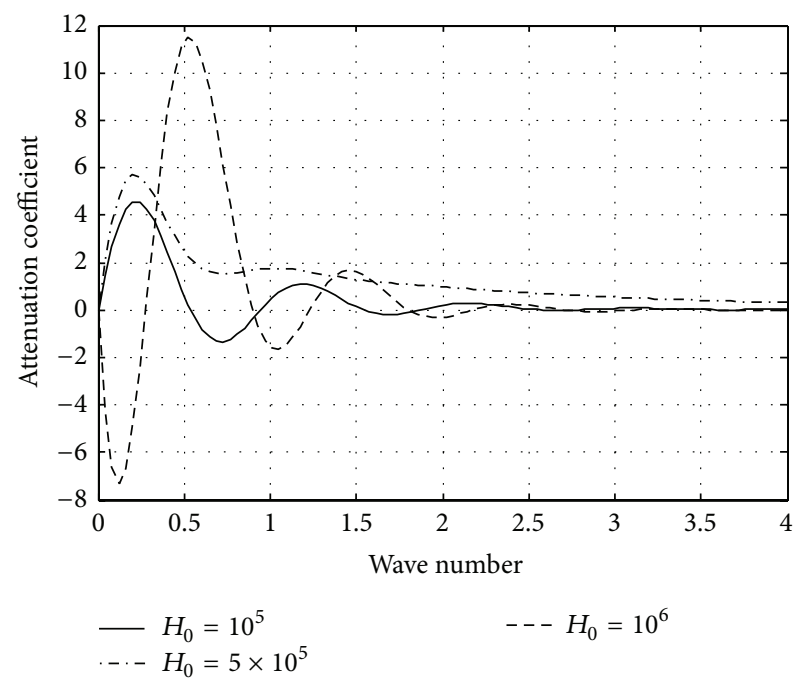

FIGURE 11: Variation of the attenuation coefficient with respect to the wave number with variation of the magnetic field.

than the corresponding values for small values of the wave number and inverses to the largest values for the large values of the wave number. Figures 10 and 11 make obvious the phase velocity and attenuation coefficients variations with respect to the wave number with variation of the magnetic field; it is concluded that the phase velocity begins from 1.2 but the attenuation coefficient begins from zero if the wave number equals zero and tends to zero if the wave number tends to infinity; also, it appears that they increase and decrease periodically with an increasing of the wave number and magnetic field that indicate the interruption of the phase velocity and attenuation coefficient with the largest values of the wave number and magnetic field that agree with the practical results. From Figure 12, it is concluded that the displacement component takes various values for zeros value of the wave number and tends to zero if the wave 


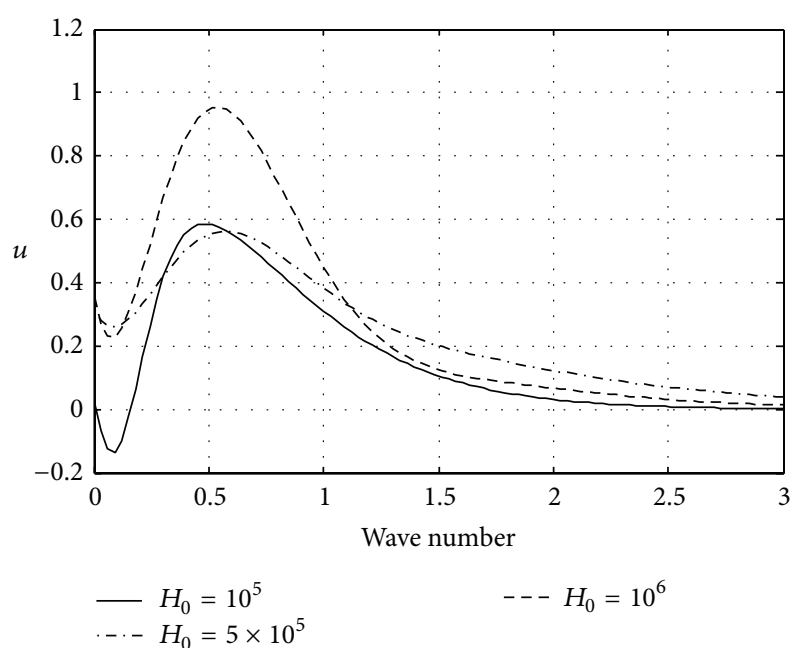

FIGURE 12: Variation of the displacement $u$ with respect to the wave number with variation of magnetic field.

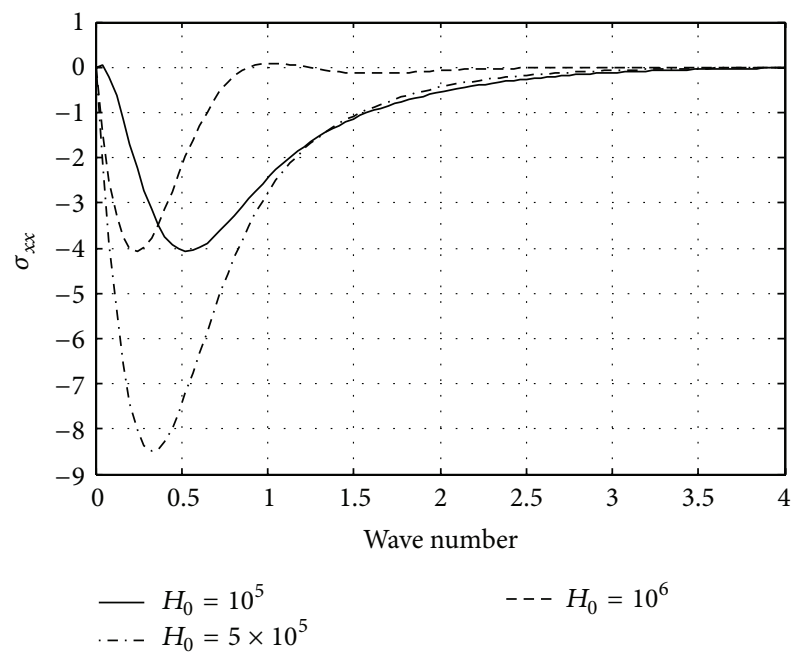

FIGURE 13: Variation of shear stress $\sigma_{x x}$ with respect to the wave number with variation of the magnetic field.

number tends to infinity; also, it is shown that it increases and decreases with an increasing of the wave number and magnetic field.

Figures 13 and 14 display the variation of the normal and shear stresses with respect to the wave number with variation of the magnetic field; it appears that it begins from zero for zeros value of the wave number and tends to zero if the wave number tends to infinity; also, it is shown that the normal stress decreases and then increases arriving to zero with the large values of the wave number and magnetic field. It is seen that the shear stress increases and periodically decreases with the variation of the wave number and magnetic field and begins from zero at zeros value of the wave number and tends again to zero if the wave number tends to infinity. Figure 15 dispatches the variation of the temperature with various values of the wave number and magnetic field; we can show that it begins from zero for zeros wave number

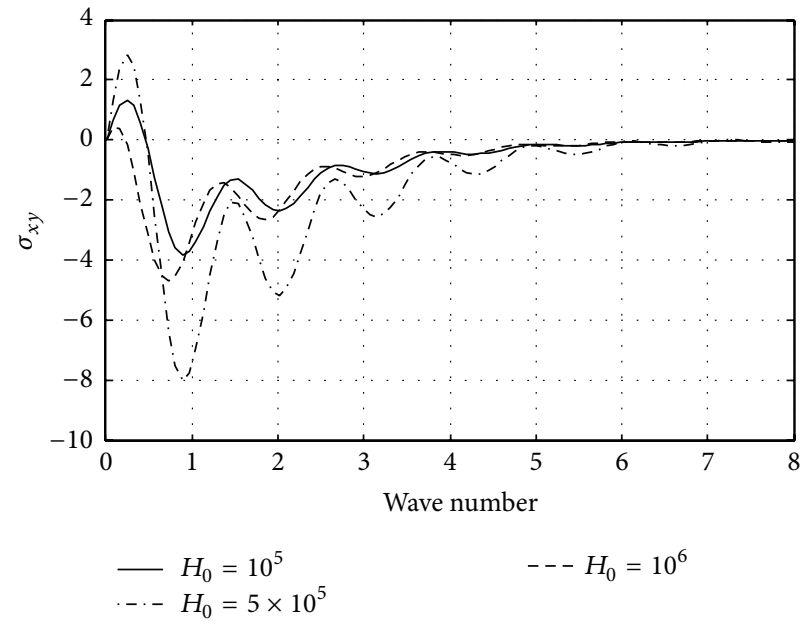

FIGURE 14: Variation of shear stress $\sigma_{x y}$ with respect to the wave number with variation of the magnetic field.

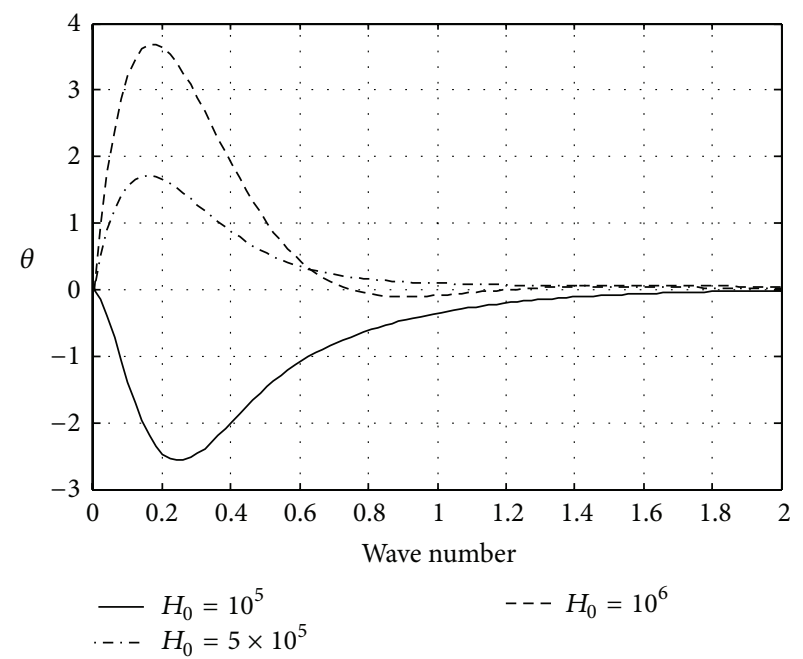

FIGURE 15: Variation of the temperature $\theta$ with respect to the wave number with variation of magnetic field.

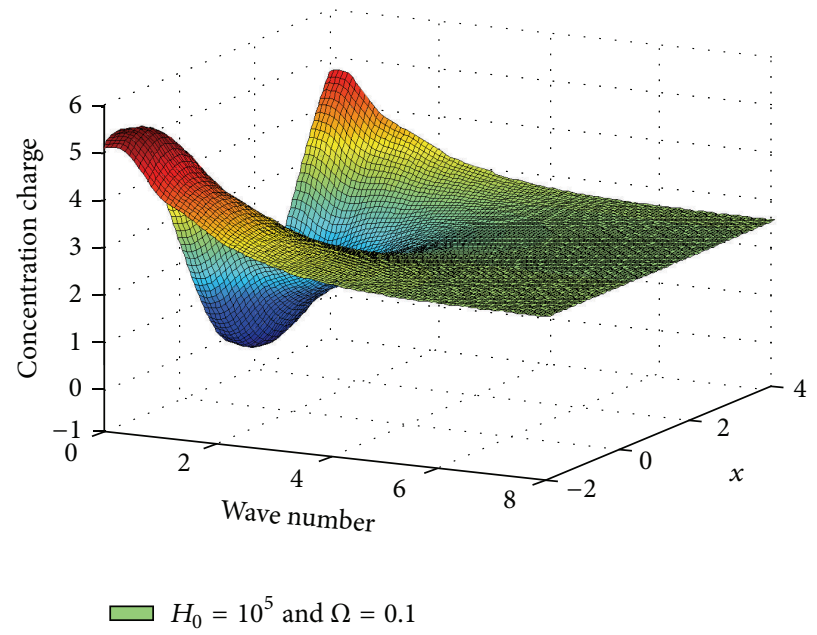

FIGURE 16: Variation of the concentration charge with respect to the wave number and $x$-axis. 


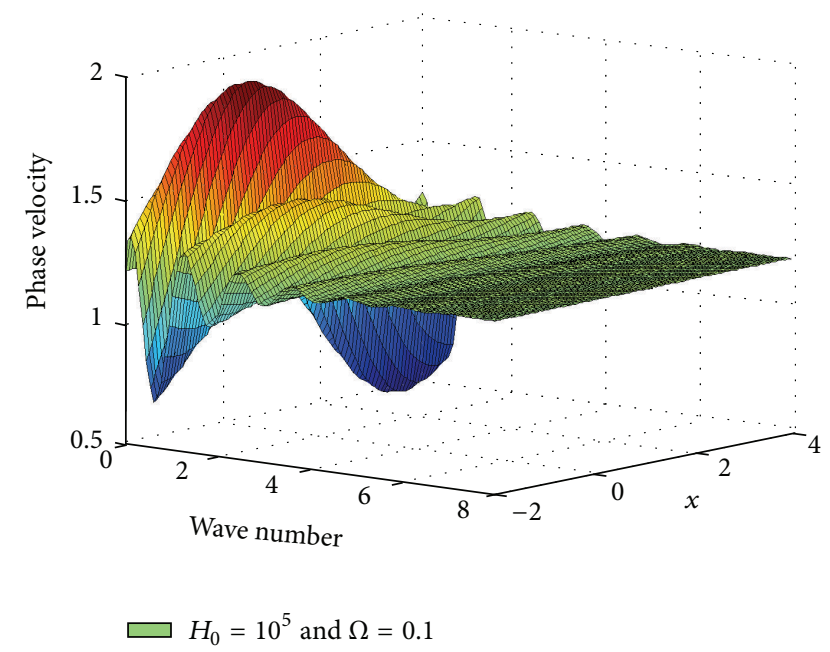

FIGURE 17: Variation of the phase velocity with respect to the wave number and $x$-axis.

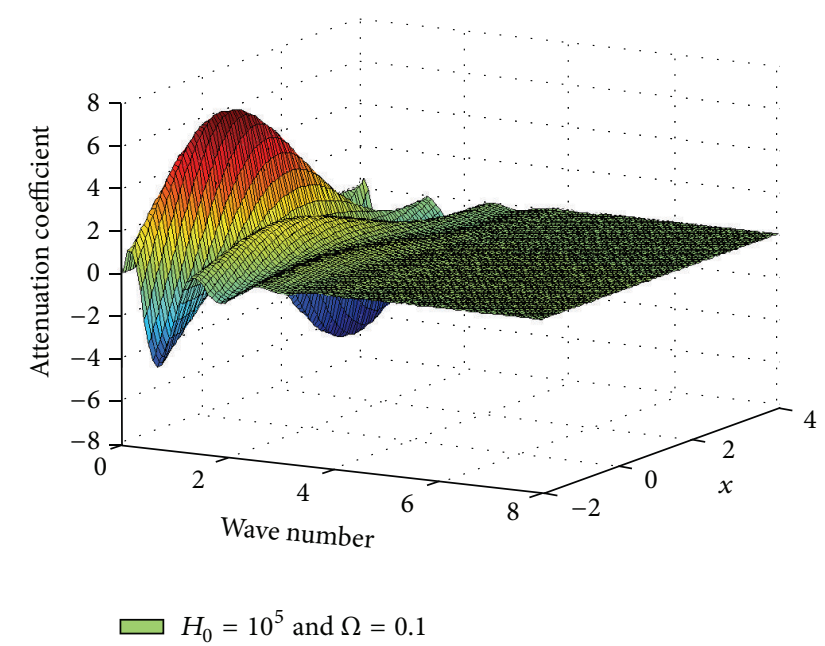

FIGURE 18: Variation of the attenuation coefficient with respect to the wave number and $x$-axis.

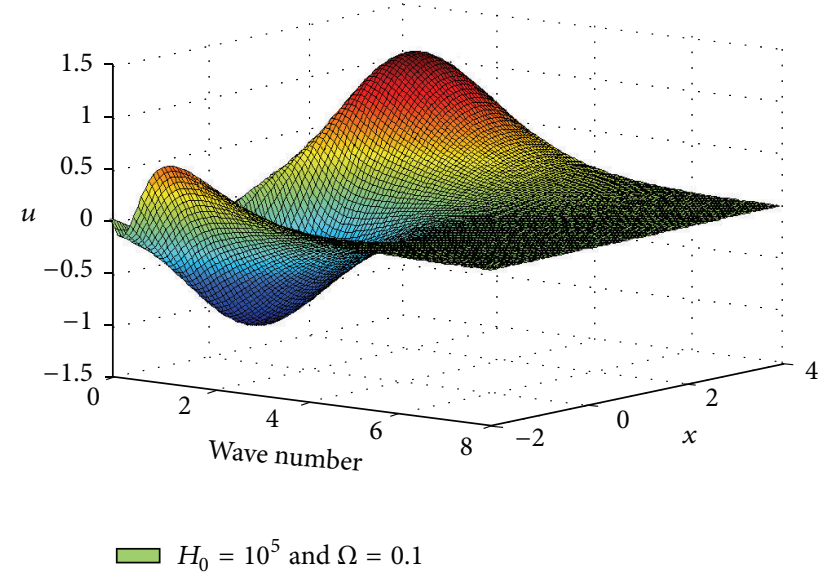

FIGURE 19: Variation of displacement $u$ with respect to the wave number and $x$-axis.

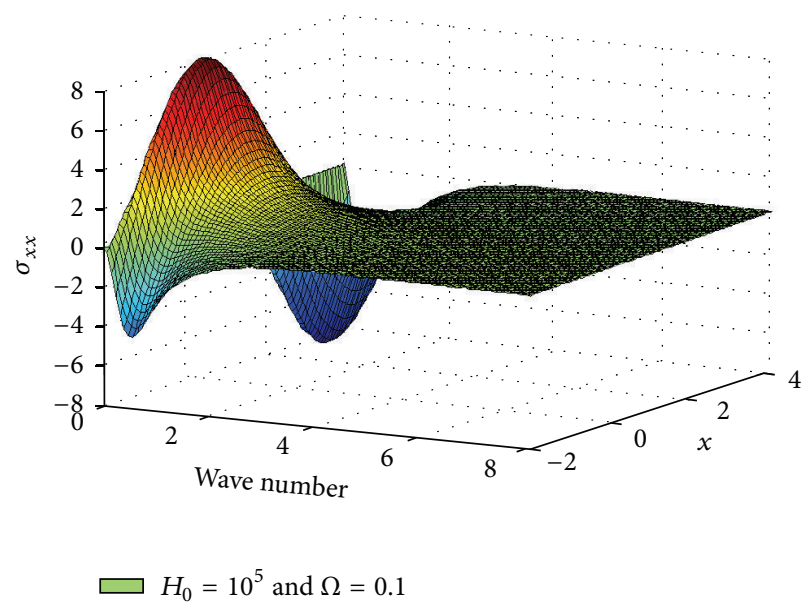

FIGURE 20: Variation of the stress $\sigma_{x x}$ with respect to the wave number and $x$-axis.

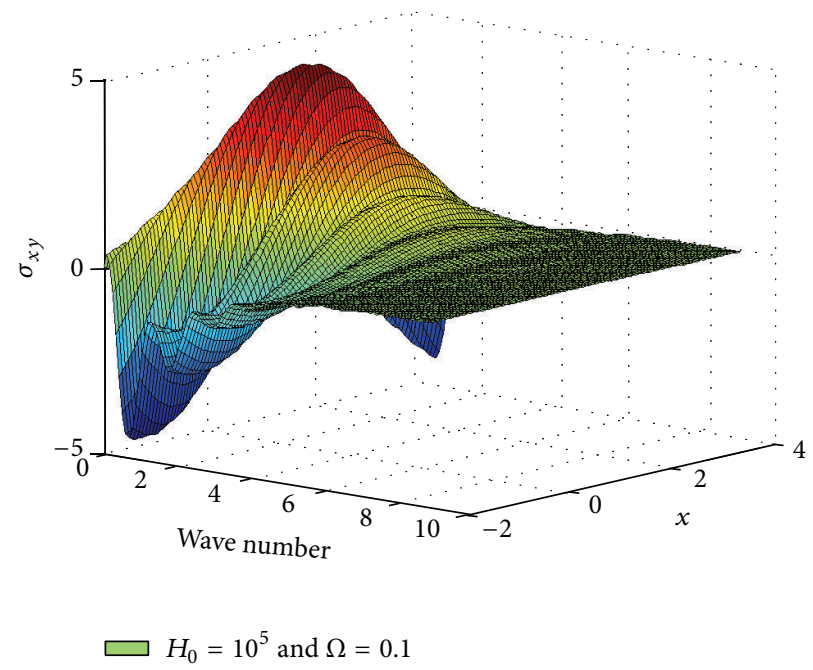

FIGURE 21: Variation of the stress $\sigma_{x y}$ with respect to the wave number and $x$-axis.

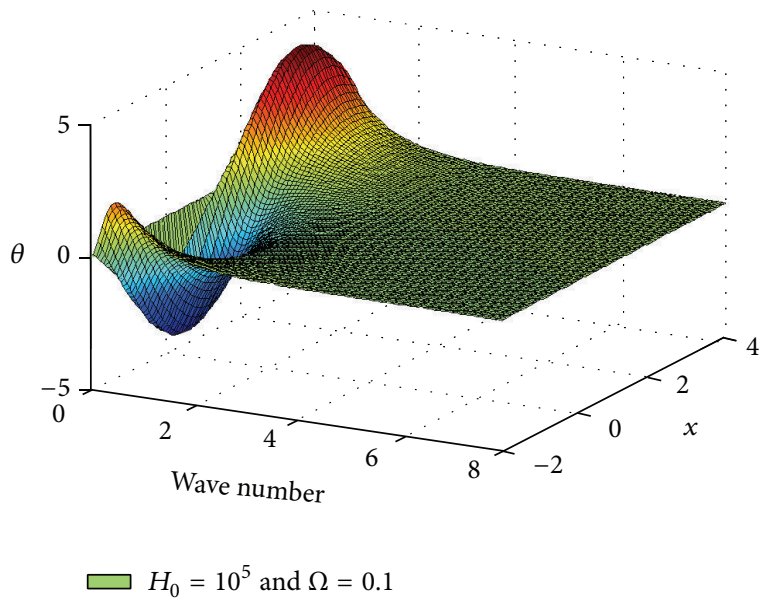

FIGURE 22: Variation of the temperature with respect to the wave number and $x$-axis. 
and tends to zero for infinity wave number and increases and decreases with variation of the wave number and magnetic field.

Totally, it is clear that the temperature decreases with increasing rotation values that physically indicates the negative influence of rotation on the temperature that takes into consideration engineering and structures.

Figures 16-22 display the 3D variation on the concentration change, phase velocity, attenuation coefficient, displacement, stresses components, and temperature, respectively, with respect to the wave number and $x$-axis if $H_{o}=10^{5}$ and $\Omega=0.1$

It is obvious from Figure 16 that the concentration change decreases to arrive to zero with the increased values of the wave number; it increases and decreases to its minimum value and after that increases with an increasing of $x$-axis. From Figures 17 and 18, it appears that the phase velocity begins from 1.2 but the attenuation coefficient begins from zero if the wave number equals zero and tends to zero if the wave number tends to infinity; also, it appears that it increases and decreases periodically and arrives to zero with an increasing of $x$-axis and the wave number. Figure 19 shows that the displacement decreases, increases periodically with the variation of the wave number, and tends to zero as the wave number tends to infinity. It appears that the displacement decreases and increases with the increased values of $x$-axis.

From Figure 20, we concluded that the normal stress decreases and then increases arriving to zero as the wave number tends to zero and increases with the small values of $x$-axis and after that decreases to tend to zero. Figure 21 shows that the shear stress component increases and decreases periodically with the variation of the wave number but increases and decreases with an increasing of $x$-axis.

Finally, it appears that the temperature increases and decreases arrive to zero as the wave number tends to infinity but decreases with an increasing of the small $x$-axis and after that increases.

\section{Concluding Remarks}

Surface waves at imperfect boundary between isotropic elastic layer of finite thickness and isotropic thermodiffusive elastic half-space with magnetic field, stiffness, and rotation with two thermal relaxation times (GL) model are illustrated. The secular equation in compact form has been derived. The concentration change, phase velocity, attenuation coefficient, displacement, stresses, and temperature are displayed graphically. The amplitudes of displacements, temperature, and concentration are computed at the free plane boundary and presented graphically. Specific loss of energy is obtained and depicted graphically.

The analysis to be carried will be useful in the design and construction of rotating sensors, engineering, structures, and surface acoustic waves devices and the following remarks have been concluded.

(1) If there is no rotation, small wave number takes larger values than in the presence of rotation and large values of the wave number.
(2) The phase velocity, attenuation coefficient, displacement, stresses components, and temperature begin from zero for zeros value of the wave number and tend to zero if the wave number tends to infinity; also, it is seen that they increase and decrease periodically with an increasing of the wave number and rotation and magnetic field.

(3) The temperature decreases with increasing rotation values that physically indicates the negative influence of rotation on the temperature that takes into consideration engineering and structures and acoustic and rotating sensors.

\section{Appendices}

\section{A. Constant of (28)}

Consider the following:

$$
\begin{aligned}
& h_{1}=c^{2}\left(1+\Lambda^{2}\right), \quad h_{2}=i \omega^{-1} c \tau_{11}, \quad h_{3}=i \omega^{-1} c \tau_{21} \text {, } \\
& h_{5}=\chi_{2} c, \quad h_{7}=-i \omega^{-1} c^{2} \chi_{1} \tau_{10} \\
& l_{1}=2 i \Lambda c^{2}, \quad l_{2}=i \omega^{-1} c \tau_{11}, \quad l_{3}=-i \omega^{-1} c \tau_{21} q_{3}^{*} \text {, } \\
& l_{4}=\omega^{-2} c^{3}, \quad l_{1}^{*}=1-i \omega^{-1} c^{2} \tau_{20}, \\
& d_{1}=\left(l_{1}^{*}\left(l_{4}-l_{3}\right)+h_{7} l_{2}\right), \quad d_{2}=\left(l_{4}+h_{7} l_{2}-l_{3} l_{1}^{*}-l_{3}\right), \\
& d_{3}=\left(l_{4} h_{5}-h_{7} q_{1}^{*}-h_{5} l_{3}\right), \\
& d_{4}=\left(l_{2} h_{5}+l_{1}^{*} q_{1}^{*}\right), \quad d_{5}=\left(l_{3} h_{5}+h_{7} q_{1}^{*}\right), \\
& d_{6}=\left(l_{2} h_{5}+l_{1}^{*} q_{1}^{*}+q_{1}^{*}\right) \text {, } \\
& d_{7}=\left(l_{3}\left(1+R_{H}^{2}\right)+h_{3} q_{1}^{*}\right), \quad d_{8}=\left(\delta_{2} d_{2}+h_{2} d_{5}\right), \\
& d_{9}=\left(h_{3} q_{1}^{*}+\delta_{2}\right) l_{3}, \\
& f_{1}=d_{2}-\left(\delta_{1}-h_{1}\right) l_{3}+\left(h_{2} d_{5}-h_{3} d_{6}\right) \delta_{1}, \\
& f_{2}=\delta_{2} d_{9}, \quad f_{3}=\left(1-h_{1}\right) d_{7}-h_{3} q_{1}^{*}-h_{3} \delta_{2} q_{1}^{*}, \\
& f_{4}=\left(d_{1}+d_{2}\left(\delta_{1}-h_{1}\right)+h_{2} d_{3}-h_{3} d_{4}\right) \delta_{1}, \\
& f_{5}=\delta_{2}^{2}\left(d_{8}-h_{3} d_{6}\right), \\
& f_{7}=h_{3}\left(\left(\delta_{1}-h_{1}\right) q_{1}^{*}-d_{6}+d_{6} \delta_{2}\right), \\
& f_{8}=\delta_{1}\left(\delta_{1}-h_{1}\right) d_{1}, \quad f_{9}=\left(\delta_{2} d_{1}-h_{2} d_{3}-d_{4} h_{3}\right) \delta_{2} \text {, } \\
& g_{1}=\delta_{2} d_{3}+\left(\delta_{1}-h_{1}\right) d_{5}-d_{3}, \\
& g_{2}=\left(\delta_{1}-h_{1}\right) d_{6}+d_{4}-\delta_{2} d_{4}, \\
& g_{3}=\left(1-h_{1}\right)\left(\delta_{1}-h_{1}\right), \quad g_{4}=l_{1}^{2} d_{1} \text {, } \\
& g_{5}=h_{2}\left(\delta_{1}-h_{1}\right), \quad g_{6}=h_{3}\left(\delta_{1}-h_{1}\right), \\
& A^{*}=\frac{-\left(f_{1}+f_{2}+f_{3}\right)}{\delta_{1} d_{7}},
\end{aligned}
$$




$$
\begin{gathered}
B^{*}=\frac{-\left(1-h_{1}\right) f_{1}+f_{4} \delta_{1}-f_{5}-f_{7}-l_{3} l_{1}^{2}}{\delta_{1} d_{7}}, \\
C^{*}=-\frac{\left(1-h_{1}\right) f_{4}-f_{9}-h_{2} g_{1}-h_{3} g_{2}+f_{8}+l_{1}^{2} d_{2}}{\delta_{1} d_{7}}, \\
D^{*}=-\frac{\left[d_{1} g_{3}+g_{4}-d_{3} g_{5}-d_{4} g_{6}\right]}{\delta_{1} d_{7}} .
\end{gathered}
$$

\section{B. Constant of (29)}

Consider the following:

$$
\begin{gathered}
n_{1 p}=-\left[\left(\Lambda_{1}+i \Lambda_{2} m_{p}-\Lambda_{3} m_{p}^{2}-i \Lambda_{4} m_{p}^{3}\right.\right. \\
\left.\left.-\Lambda_{5} m_{p}^{4}-i \Lambda_{9} m_{p}^{5}\right) \times\left(E_{p}^{*}\right)^{-1}\right], \\
n_{2 p}=\left[\frac{\Lambda_{7}+\Lambda_{8} m_{p}+\Lambda_{9} m_{p}^{2}-\Lambda_{10} m_{p}^{3}+\Lambda_{11} m_{p}^{4}}{E_{p}^{*}}\right], \\
n_{3 p}=-\left[\left(\Lambda_{12}-\Lambda_{13} m_{p}+\Lambda_{14} m_{p}^{2}\right.\right. \\
-\Lambda_{15} m_{p}^{3}+\Lambda_{16} m_{p}^{4}-\Lambda_{17} m_{p}^{5} \\
\left.\left.+\Lambda_{18} m_{p}^{6}\right) \times\left(E_{p}^{*}\right)^{-1}\right] \\
E_{p}^{*}=G_{1}-G_{2} m_{p}^{2}-G_{3} m_{p}^{6}+G m_{p}^{8}, \quad p=1,2,3,4,
\end{gathered}
$$

where

$$
\begin{gathered}
G_{1}=\left(\delta_{1}-h_{1}\right) d_{1}, \\
G_{2}=\left(d_{1}+\left(\delta_{1}-h_{1}\right) d_{2}-h_{2} d_{3}-h_{3} d_{4}\right), \\
G_{3}=\left(d_{2}-\left(\delta_{1}-h_{1}\right) l_{3}+h_{2} d_{5}-h_{3} d_{6}\right), \\
G_{4}=d_{7}, \quad \Lambda_{1}=l_{1} d_{1}, \\
\Lambda_{2}=\left(\delta_{2}+R_{H}^{2}\right) d_{1}-h_{2} d_{3}-h_{3} d_{4}, \\
\Lambda_{3}=l_{1} d_{2}, \quad \Lambda_{4}=d_{8}-h_{3} d_{6}, \\
\Lambda_{5}=-l_{1} l_{3}, \quad \Lambda_{6}=d_{9}, \\
\Lambda_{7}=-\left(\delta_{1}-h_{1}\right) d_{3}, \quad \Lambda_{8}=l_{1} d_{3}, \\
\Lambda_{9}=\left(\delta_{2} d_{3}+\left(\delta_{1}-h_{1}\right) d_{5}-d_{3}\right), \\
\Lambda_{10}=l_{1} d_{5}, \quad \Lambda_{11}=d_{5}-\delta_{2} d_{5}, \\
\Lambda_{12}=\left(\delta_{1}-h_{1}\right) d_{4}, \quad \Lambda_{13}=l_{1} d_{4}, \\
\Lambda_{14}=d_{4}+\left(\delta_{1}-h_{1}\right) d_{6}-\delta_{2} d_{4}, \\
\Lambda_{15}=l_{1} d_{6}, \quad \Lambda_{16}=d_{6}+\left(\delta_{1}-h_{1}\right) q_{1}^{*}-\delta_{2} d_{6}, \\
\Lambda_{17}=q_{1}^{*} l_{1}, \quad \Lambda_{18}=q_{1}^{*}\left(1-\delta_{2}\right) .
\end{gathered}
$$

\section{Determinant Elements of (36)}

Consider the following:

$$
\begin{aligned}
& R_{14}=R_{15}=R_{16}=R_{17}=R_{21}=R_{22}=R_{23}=R_{31} \\
& =R_{32}=R_{33}=R_{42}=R_{51}=R_{53}=0, \\
& R_{11}=w_{8}, \quad R_{12}=-y_{7}, \quad R_{13}=w_{7}, \\
& R_{24}=b_{2}, \quad R_{25}=b_{1}, \quad R_{26}=b_{3}, \quad R_{27}=b_{4} \text {, } \\
& R_{34}=q_{2}, \quad R_{35}=q_{1}, \quad R_{36}=q_{3}, \\
& R_{37}=q_{4}, \quad R_{41}=\left(b_{5}+b_{9}\right), \quad R_{43}=-b_{6}, \\
& R_{44}=J_{2}, \quad R_{45}=J_{1}, \quad R_{46}=J_{3}, \\
& R_{47}=J_{4}, \quad R_{52}=b_{7}, \quad R_{54}=s_{2}^{*} \text {, } \\
& R_{55}=s_{1}^{*}, \quad R_{56}=s_{3}^{*}, \quad R_{57}=s_{4}^{*}, \\
& R_{16}=\left[b_{5}+b_{9}\right], \quad R_{62}=-i \xi \frac{k_{n}}{\beta_{1} T_{0}}, \quad R_{63}=-b_{6} \text {, } \\
& R_{64}=r_{2}, \quad R_{65}=r_{1}, \quad R_{66}=r_{3}, \\
& R_{67}=r_{4}, \quad R_{71}=-i \xi \frac{k_{1}}{\beta_{1} T_{0}}, \quad R_{72}=b_{7}, \\
& R_{73}=\xi m_{6} \frac{k_{1}}{\beta_{1} T_{0}}, \quad R_{74}=k_{1}, \\
& R_{75}=k_{1}, \quad R_{76}=k_{1}, \quad R_{77}=k_{1}, \\
& R_{11}^{*}=y_{8}, \quad R_{41}^{*}=0, \quad R_{51}^{*}=b_{8} \text {, } \\
& R_{61}^{*}=-\xi m_{5} \frac{k_{n}}{\beta_{1} T_{0}}, \quad R_{71}^{*}=-b_{8}, \quad R_{12}^{*}=w_{8}, \\
& R_{52}^{*}=0, \quad R_{42}^{*}=\left(b_{5}+b_{9}\right)=R_{62}^{*}, \quad R_{72}^{*}=-i \xi \frac{k_{1}}{\beta_{1} T_{0}}, \\
& R_{13}^{*}=-y_{7}, \quad R_{43}^{*}=0, \quad R_{53}^{*}=-b_{7}, \\
& R_{63}^{*}=-i \xi \frac{k_{n}}{\beta_{1} T_{0}}, \quad R_{73}^{*}=b_{7}, \\
& w_{8}=b_{8} \sin \left(\xi m_{5} H\right), \quad w_{5}=b_{5} \cos \left(\xi m_{5} H\right), \\
& w_{7}=b_{7} \sin \left(\xi m_{6} H\right), \quad w_{6}=b_{6} \sin \left(\xi m_{6} H\right), \\
& b_{8}=2 i m_{5} \Gamma_{1}^{e}, \quad b_{7}=\left(1-m_{6}^{2}\right) \Gamma_{1}^{e}, \\
& b_{5}=\frac{\left(\left(\lambda^{e}+\mu_{e} H_{Y}^{2}\right)\left(1+m_{5}^{2}\right)+2 \mu^{e} m_{5}^{2}\right) \xi^{2}}{a_{1} T_{0}} \text {, } \\
& b_{6}=\frac{2 i \mu^{e} m_{6} \xi}{a_{1} T_{0}}, \\
& y_{7}=-b_{7} \cos \left(\xi m_{6} H\right), \quad y_{5}=b_{5} \sin \left(\xi m_{5} H\right) \text {, } \\
& y_{8}=b_{8} \cos \left(\xi m_{5} H\right), \quad y_{6}=b_{6} \cos \left(\xi m_{6} H\right),
\end{aligned}
$$




$$
\begin{gathered}
J_{p}=i \Gamma_{2} \xi-\Gamma_{3} n_{2 p}-\Gamma_{4} n_{3 p}-\Gamma_{5} \xi n_{1 p} m_{p} \\
+\frac{\mu_{e} H_{Y}^{2}}{\beta_{1} T_{0}} i \xi\left(i-n_{1 p} m_{p}\right), \\
b_{p}=n_{2 p} m_{p}, \quad q_{p}=n_{3 p} m_{p}, \\
r_{p}=k_{n} n_{1 p}, \quad \Gamma_{5}=\frac{\lambda+2 \mu}{a_{1} T_{0}}, \quad \Gamma_{2}=\frac{\lambda}{\beta_{1} T_{0}}, \\
\Gamma_{3}=\frac{\rho v_{1}^{2} \tau_{11}}{\beta_{1} T_{0}}, \quad \Gamma_{4}=\frac{\rho v_{1}^{2} \tau_{21}}{\beta_{1} T_{0}}, \\
\Gamma_{1}^{e}=\frac{\mu^{e}}{\beta_{1} T_{0}}, \quad \Gamma_{1}=\frac{\mu}{\beta_{1} T_{0}}, \\
s_{p}^{*}=\left[i \xi n_{1 p}-\xi m_{p}\right] \Gamma_{1} .
\end{gathered}
$$

\section{D. $F_{P}^{*}(P=1,2,3,4)$ Given in (46)}

Consider the following:

$$
\begin{aligned}
& F_{1}^{*}=q_{2}\left(J_{3} s_{4}^{*}-J_{4} s_{3}^{*}\right)-q_{3}\left(J_{2} s_{4}^{*}-J_{4} s_{2}^{*}\right)+q_{4}\left(J_{2} s_{3}^{*}-J_{3} s_{2}^{*}\right), \\
& F_{2}^{*}=q_{1}\left(J_{3} s_{4}^{*}-J_{4} s_{3}^{*}\right)-q_{3}\left(J_{1} s_{4}^{*}-J_{4} s_{1}^{*}\right)+q_{4}\left(J_{1} s_{3}^{*}-J_{3} s_{1}^{*}\right), \\
& F_{3}^{*}=q_{1}\left(J_{2} s_{4}^{*}-J_{4} s_{2}^{*}\right)-q_{2}\left(J_{1} s_{4}^{*}-J_{1} s_{2}^{*}\right)+q_{4}\left(J_{2} s_{1}^{*}-J_{1} s_{2}^{*}\right), \\
& F_{1}^{*}=q_{1}\left(J_{2} s_{3}^{*}-J_{3} s_{2}^{*}\right)-q_{2}\left(J_{1} s_{3}^{*}-J_{3} s_{1}^{*}\right)+q_{3}\left(J_{1} s_{2}^{*}-J_{2} s_{3}^{*}\right) .
\end{aligned}
$$

\section{Conflict of Interests}

The authors declare that there is no conflict of interests regarding the publication of this paper.

\section{References}

[1] L. Knopoff, "The interaction between elastic wave motion and a magnetic field in electrical conductors," Journal of Geophysical Research, vol. 60, pp. 441-456, 1955.

[2] P. Chadwick, "Elastic wave propagation in a magnetic field," in Proceedings of the 9th International Congress of Applied Mechanics, vol. 7, pp. 143-153, 1957.

[3] S. Kaliski and J. Petykiewicz, "Equation of motion coupled with the field of temperature in a magnetic field involving mechanical and electrical relaxation for anisotropic bodies," Proceedings of Vibration Problems, vol. 4, article 1, 1959.

[4] H. Y. Yu, Y. N. Wei, and F. P. Chiang, "Load transfer at imperfect interfaces-dislocation-like model," International Journal of Engineering Science, vol. 40, no. 14, pp. 1647-1662, 2002.

[5] H. Y. Yu, "A new dislocation-like model for imperfect interfaces and their effect on load transfer," Composites Part A: Applied Science and Manufacturing, vol. 29, no. 9-10, pp. 1057-1062, 1998.

[6] Y. Benveniste, "The effective mechanical behaviour of composite materials with imperfect contact between the constituents," Mechanics of Materials, vol. 4, no. 2, pp. 197-208, 1985.

[7] H. W. Lord and Y. Shulman, "A generalized dynamical theory of thermoelasticity," Journal of the Mechanics and Physics of Solids, vol. 15, no. 5, pp. 299-309, 1967.
[8] A. E. Green and K. A. Lindsay, “Thermoelasticity," Journal of Elasticity, vol. 2, no. 1, pp. 1-7, 1972.

[9] R. B. Hetnarski and J. Ignaczak, "Generalized thermoelasticity," Journal of Thermal Stresses, vol. 22, no. 4-5, pp. 451-476, 1999.

[10] W. Nowacki, "Dynamical problem of thermoelastic diffusion in solid-1," Bulletin of Polish Academy of Sciences Series, Science and Technology, vol. 22, pp. 55-64, 1974.

[11] W. Nowacki, "Dynamical problem of thermoelastic diffusion in solid-11," Bulletin of Polish Academy of Sciences Series, Science and Technology, vol. 22, pp. 129-135, 1974.

[12] W. Nowacki, "Dynamical problem of thermoelastic diffusion in solid-111," Bulletin of Polish Academy of Sciences Series, Science and Technology, vol. 22, pp. 275-276, 1974.

[13] W. Nowacki, "Dynamic problems of thermodiffusion in elastic solids," Proceedings of Vibration Problems, vol. 15, pp. 105-128, 1974.

[14] Z. S. Olesiak and Y. A. Pyryev, "A coupled quasi-stationary problem of thermodiffusion for an elastic cylinder," International Journal of Engineering Science, vol. 33, no. 6, pp. 773-780, 1995.

[15] H. H. Sherief, F. A. Hamza, and H. A. Saleh, "The theory of generalized thermoelastic diffusion," International Journal of Engineering Science, vol. 42, no. 5-6, pp. 591-608, 2004.

[16] H. H. Sherief and H. A. Saleh, "A half-space problem in the theory of generalized thermoelastic diffusion," International Journal of Solids and Structures, vol. 42, no. 15, pp. 4484-4493, 2005.

[17] B. Singh, "Reflection of P and SV waves from free surface of an elastic solid with generalized thermodiffusion," Journal of Earth System Science, vol. 114, no. 2, pp. 159-168, 2005.

[18] B. Singh, "Reflection of SV waves from the free surface of an elastic solid in generalized thermoelastic diffusion," Journal of Sound and Vibration, vol. 291, no. 3-5, pp. 764-778, 2006.

[19] M. Aouadi, "Variable electrical and thermal conductivity in the theory of generalized thermoelastic diffusion," Zeitschrift für Angewandte Mathematik und Physik, vol. 57, no. 2, pp. 351-367, 2006.

[20] M. Aouadi, "A generalized thermoelastic diffusion problem for an infinitely long solid cylinder," International Journal of Mathematics and Mathematical Sciences, vol. 2006, Article ID 25976, 15 pages, 2006.

[21] M. Aouadi, "A problem for an infinite elastic body with a spherical cavity in the theory of generalized thermoelastic diffusion," International Journal of Solids and Structures, vol. 44, no. 17, pp. 5711-5722, 2007.

[22] J. N. Sharma and V. Walia, "Effect of rotation on Rayleigh waves in piezothermoelastic half space," International Journal of Solids and Structures, vol. 44, no. 3-4, pp. 1060-1072, 2007.

[23] J. N. Sharma, V. Walia, and S. K Gupta, "Effect of rotation and thermal relaxation on Rayleigh waves in piezothermoelastic half space," International Journal of Mechanical Sciences, vol. 50, no. 3, pp. 433-444, 2008.

[24] R. Kumar and T. Kansal, "Propagation of Rayleigh waves on free surface of transversely isotropic generalized thermoelastic diffusion," Applied Mathematics and Mechanics, vol. 29, no. 11, pp. 1451-1462, 2008.

[25] R. Kumar and T. Kansal, "Propagation of Lamb waves in transversely isotropic thermoelastic diffusive plate," International Journal of Solids and Structures, vol. 45, no. 22-23, pp. 58905913, 2008.

[26] N. C. Dawn and S. K. Chakraborty, "On Rayleigh waves in Green-Lindsay's model of generalized thermoelastic media," 
Indian Journal of Pure and Applied Mathematics, vol. 20, no. 3, pp. 276-283, 1988.

[27] R. Kumar and V. Chawla, "Effect of rotation and stiffness on surface wave propagation in a elastic layer lying over a generalized thermodiffusive elastic half-space with imperfect boundary," Journal of Solid Mechanics, vol. 2, no. 1, pp. 28-42, 2010.

[28] S. M. Abo-Dahab and B. Singh, "Influences of magnetic field on wave propagation in generalized thermoelastic solid with diffusion," Archives of Mechanics, vol. 61, no. 2, pp. 121-136, 2009.

[29] S. M. Abo-Dahab, "Propagation of $P$ waves from stress-free surface elastic half-space with voids under thermal relaxation and magnetic field," Applied Mathematical Modelling, vol. 34, no. 7, pp. 1798-1806, 2010.

[30] S. M. Abo-Dahab, R. A. Mohamed, and B. Singh, "Rotation and magnetic field effects on $\mathrm{P}$ wave reflection from stressfree surface elastic half-space with voids under one thermal relaxation time," Journal of Vibration and Control, vol. 17, no. 12, pp. 1827-1839, 2011.

[31] A. M. Abd-Alla, S. M. Abo-Dahab, and F. S. Bayones, "Effect of the rotation on an infinite generalized magneto-thermoelastic diffusion body with a spherical cavity," International Review of Physics, vol. 5, no. 4, pp. 171-181, 2011.

[32] A. M. Abd-Alla and S. M. Abo-Dahab, "Effect of rotation and initial stress on an infinite generalized magneto-thermoelastic diffusion body with a spherical cavity," Journal of Thermal Stresses, vol. 35, no. 10, pp. 892-912, 2012.

[33] K. E. Bullen, An Introduction of the Theory of Seismology, Cambridge University Press, Cambridge, UK, 1963.

[34] W. M. Ewing, W. S. Jardetzky, and F. Press, Elastic Layers in Layered Media, McGraw-Hill, New York, NY, USA, 1957.

[35] H. Kolsky, Stress Waves in Solids, Clarendon Press, Oxford, UK, Dover Press, New York, NY, USA, 1963. 


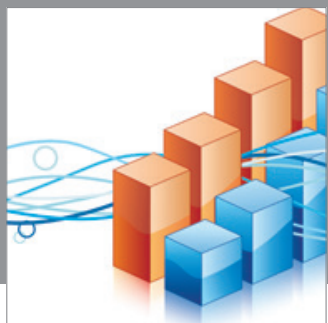

Advances in

Operations Research

mansans

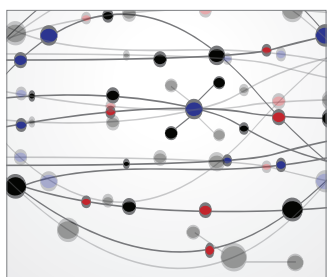

The Scientific World Journal
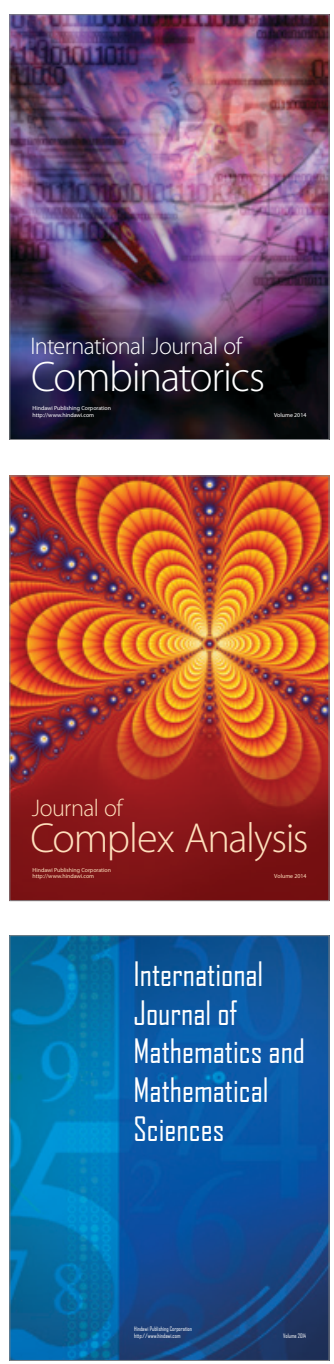
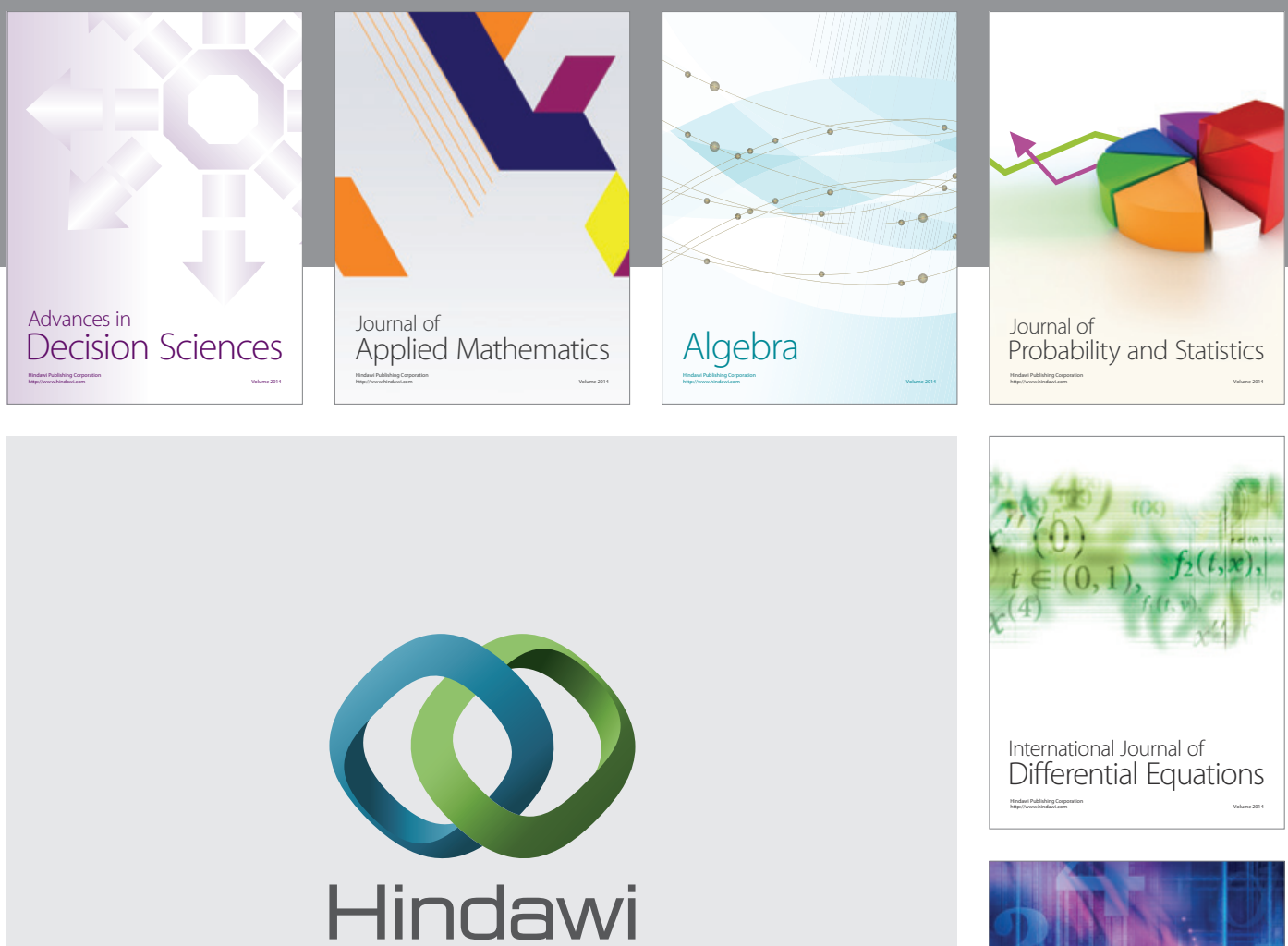

Submit your manuscripts at http://www.hindawi.com
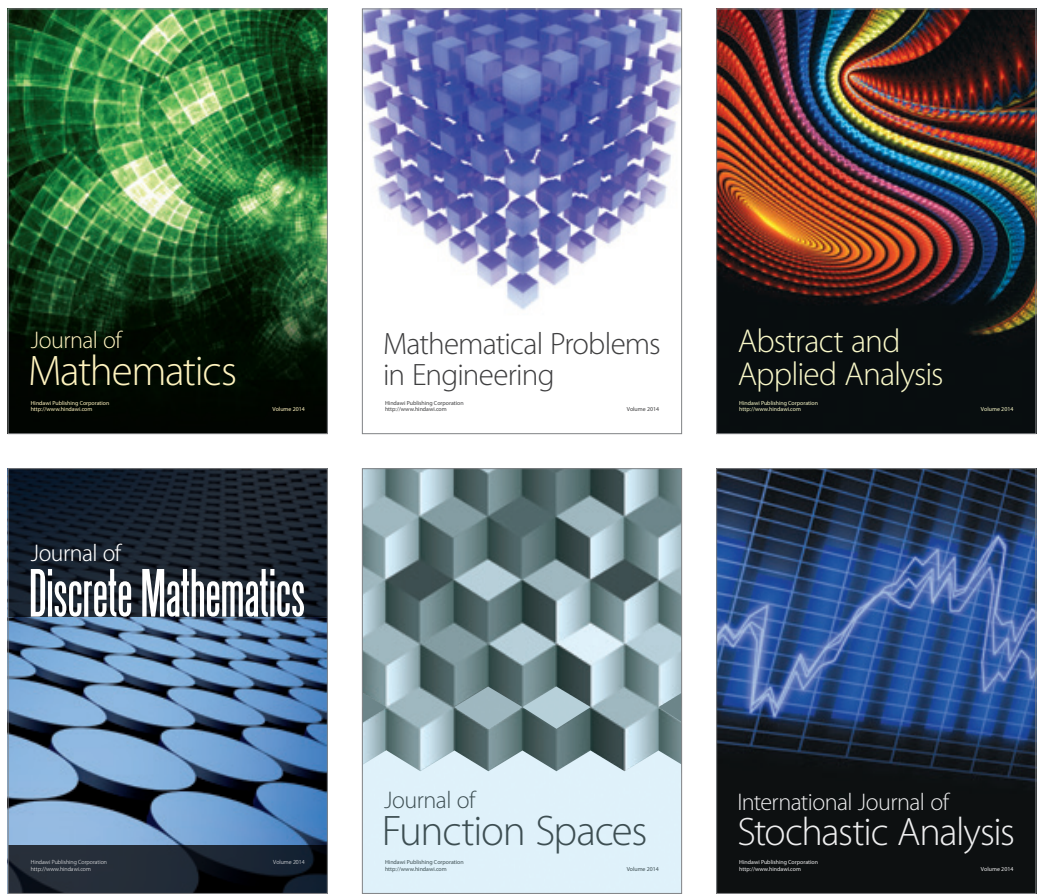

Journal of

Function Spaces

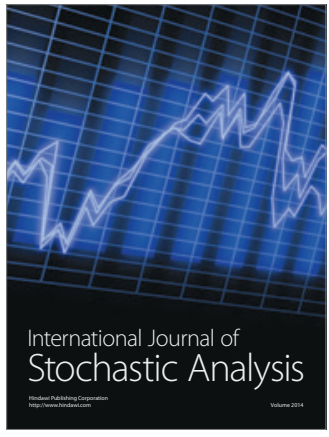

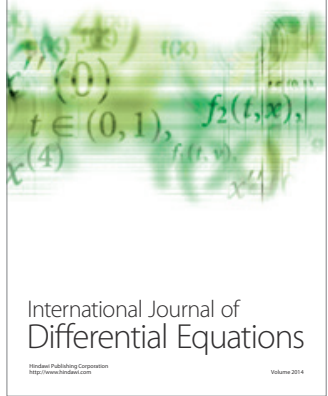
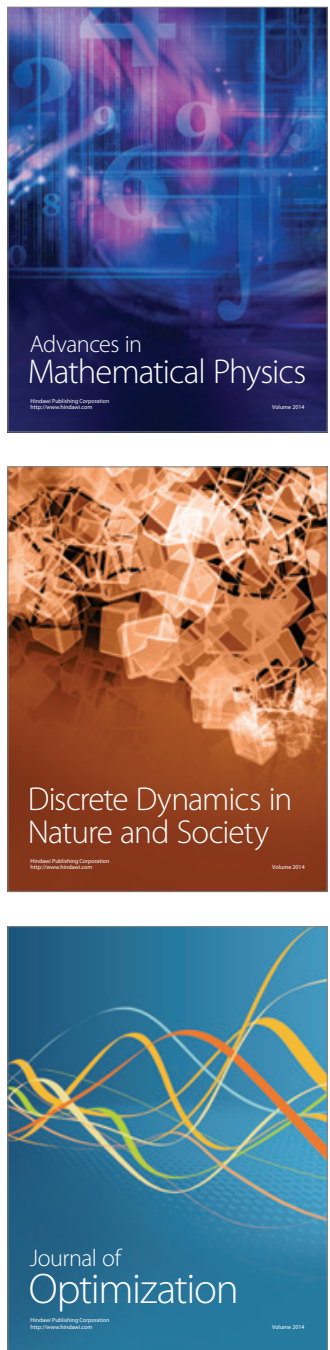